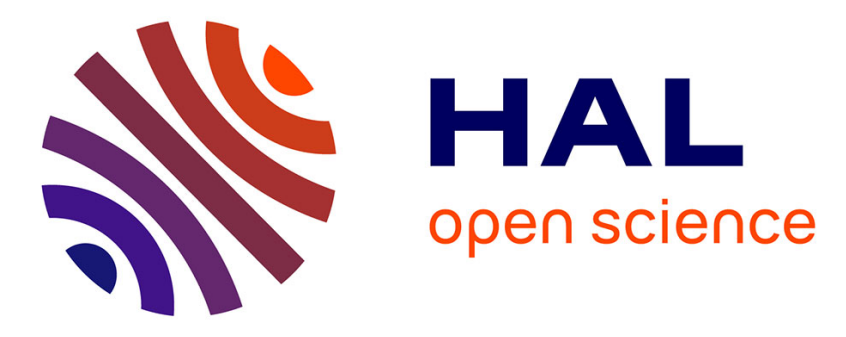

\title{
Energy Envelope and Attenuation Characteristics of High-Frequency (HF) and Very-High-Frequency (VF) Martian Events
}

Sabrina Menina, Ludovic Margerin, Taïchi Kawamura, Philippe Lognonné, Jules Marti, Mélanie Drilleau, Marie Calvet, Nicolas Compaire, Raphaël Garcia, Foivos Karakostas, et al.

\section{To cite this version:}

Sabrina Menina, Ludovic Margerin, Taïchi Kawamura, Philippe Lognonné, Jules Marti, et al.. Energy Envelope and Attenuation Characteristics of High-Frequency (HF) and Very-High-Frequency (VF) Martian Events. Bulletin of the Seismological Society of America, In press, 10.1785/0120210127. hal-03427455

\section{HAL Id: hal-03427455 \\ https://hal.science/hal-03427455}

Submitted on 13 Nov 2021

HAL is a multi-disciplinary open access archive for the deposit and dissemination of scientific research documents, whether they are published or not. The documents may come from teaching and research institutions in France or abroad, or from public or private research centers.
L'archive ouverte pluridisciplinaire $\mathbf{H A L}$, est destinée au dépôt et à la diffusion de documents scientifiques de niveau recherche, publiés ou non, émanant des établissements d'enseignement et de recherche français ou étrangers, des laboratoires publics ou privés. 


\section{Energy envelope and attenuation characteristics of}

\section{High Frequency (HF) and Very High Frequency (VF)}

\section{Martian events}

S. Menina ${ }^{1}$, L. Margerin ${ }^{2}$, T. Kawamura ${ }^{1}$, P. Lognonné ${ }^{1}$, J. Marti $^{2}$, M.

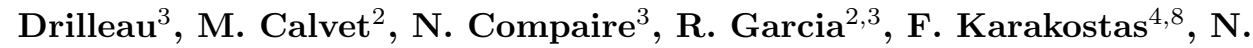

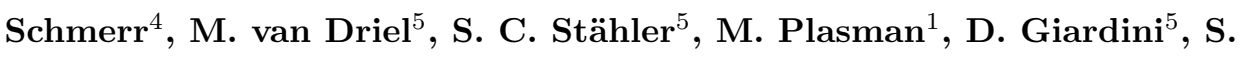

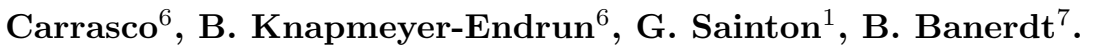

${ }^{1}$ Institut de Physique du Globe de Paris, Université de Paris, CNRS F-75005 Paris, France ${ }^{2}$ Institut de Recherche en Astrophysique et Planétologie, Université Toulouse III Paul Sabatier, CNRS, CNES, 31400 Toulouse, France

${ }^{3}$ Institut Supérieur de l'Aéronautique et de l'Espace SUPAERO, 10 Avenue Edouard Belin, 31400 Toulouse, France

${ }^{4}$ Department of Geology, University of Maryland, College Park, MD, USA ${ }^{5}$ Institute for Geophysics, ETH Zürich, Zürich, Switzerland ${ }^{6}$ Bensberg Observatory, University of Cologne, Cologne, Germany

${ }^{7}$ Jet Propulsion Laboratory, California Institute of Technology, Pasadena, CA 91109, USA

${ }^{8}$ Istituto Nazionale di Geofisica e Vulcanologia, Sezione di Bologna, Bologna, Italy

\section{Declaration of Competing Interest}

The authors acknowledge there are no conflicts of interest recorded.

Corresponding author: Sabrina Menina, menina@ipgp.fr 


\section{Abstract}

Since its deployment at the surface of Mars, the SEIS instrument of the InSight mission has detected hundreds of small magnitude seismic events. In this work, we highlight some features of two specific families: High-Frequency (HF) and Very-HighFrequency (VF) events. We characterize the shape of the energy envelopes of HF and VF events with two parameters: (1) The delay-time $t_{d}$ between the onset and the peak of the dominant arrival; (2) The quality factor $Q_{c}$ which quantifies the energy decay rate in the coda. We observe that the envelope of HF and VF events is frequency-independent. As a consequence, a single delay-time suffices to characterize envelope broadening in the $2.5-7.5 \mathrm{~Hz}$ band. The typical coda decay time is also frequency-independent as attested by the close to linear increase of $Q_{c}$ with frequency. Finally, we use elastic radiative transfer theory to perform a series of inversion of seismogram envelopes for the attenuation properties of the Martian lithosphere. The good fit between synthetic and observed envelopes confirms that multiple scattering of elastic waves released by internal sources is a plausible explanation of the events characteristics. We quantify scattering and attenuation properties of Mars and highlight the differences/similarities with the Earth and the Moon. The albedo, i.e. the contribution of scattering to the total attenuation, derived from VF events is very high which we interpret as a signature of a mostly dry medium. Our results also suggest a stratification of the scattering/attenuation properties. 


\section{Introduction}

InSight (Interior Exploration using Seismic Investigations, Geodesy and Heat Transport, Banerdt et al., 2020) has deployed on Mars a complete geophysical payload including a seismometer. Since the start of its operation, the SEIS (Seismic Experiment for Interior Structure) seismometer (Lognonné et al., 2019, 2020) recorded several hundreds of seismic events (Giardini et al., 2020). These events have been classified into two main families: high-frequency and low-frequency, depending on whether their frequency content is dominant above or below $1 \mathrm{~Hz}$ (Clinton et al., 2021). Among all the events reported by the Mars Quake Service (MQS), the majority belongs to the high frequency family, which is further divided into High-Frequency (HF) and VeryHigh Frequency (VF) event types. In the HF group, we include specific events with most of their energy in a narrow frequency band around $2.4 \mathrm{~Hz}$, for which the term "2.4 Hz events" has been coined (Clinton et al., 2021). The frequency content of relatively rare events called "Broadband" covers the range $0.1-2.5 \mathrm{~Hz}$. They are excluded from our analysis. The main seismological characteristics of HF and VF events (e.g. tentative location, temporal distribution) have been presented in detail in van Driel et al. (2021). A brief account of their findings is reported hereafter. HF and VF events are characterized by two temporally separated arrivals with a gradual beginning, a broad maximum and a long coda lasting up to 16 minutes. They can be clearly distinguished from events of type low-frequency and broadband, whose spectra fall off above 1 and $2.5 \mathrm{~Hz}$, respectively and who have clearly separated impulsive P- and S-arrivals, similar to teleseismic earthquakes. The morphology of HF and VF seismograms is reminiscent of moonquakes and high-frequency crustal earthquakes with some notable differences (Lognonné et al., 2020; van Driel et al., 2021). Compared to moonquakes, the dominant arrivals are more impulsive, the envelope broadening is less pronounced and the coda duration (typically 10 minutes) is shorter. For comparison, Latham et al. (1970) and Nakamura et al. (1982) analyzed seismic signals collected during the Apollo missions (1969-1977) and reported observations of long ringing codas lasting up 
to 2 hours. Latham et al. (1972) and Nakamura (1976) related these characteristics to the strong scattering in the heterogeneous upper lunar crust. In contrast with crustal earthquakes, HF and VF events do not exhibit clearly polarized first arrivals and their coda duration is much longer. The accrued duration of the coda is likely a consequence of both the low noise level on Mars and the slower energy decay as compared to Earth. van Driel et al. (2021) provide convincing evidence that $\mathrm{HF}$ and VF events result from the propagation of seismic waves in a low-attenuation, strongly scattering medium. In this work, we pursue the investigation of HF and VF events by characterizing in details their envelope shape and by inverting for the scattering/attenuation properties of Mars using full seismogram envelope modeling in 3-D.

It is well established that multiple-scattering hampers the identification and analysis of ballistic body-wave phases (Sato et al., 2012). As a consequence, traditional methods used in terrestrial seismology can become impractical when the near-surface is strongly heterogeneous. This is the case on the Moon, due to the presence of the megaregolith, but also in volcanic areas on Earth (Mayeda et al., 1992). Fortunately, a large amount of information on the properties and structure of the scattering layers can be extracted from the detailed analysis of seismogram envelopes. The overall shape of the envelopes can be described by two parameters. The first one is the peak delay, i.e., the delay time from the onset to the peak intensity of S-waves. It is a convenient tool to quantify the effects of strong diffraction of the waves in a heterogeneous medium. Takahashi et al. (2007) gave convincing examples of the path dependence of the peak delay in northern Japan. They found that large peak delays are correlated with strong scattering due to the medium inhomogeneity. The second parameter is the coda quality factor $Q_{c}$, which quantifies the rate of decay of the coda. $Q_{c}$ has been widely used in lithospheric or crustal attenuation studies (Aki \& Chouet, 1975; Mitchell, 1995; Sato et al., 2012). 
Besides attenuation estimation based on $Q_{c}$ measurements, several methods were proposed to evaluate directly the contributions of scattering and absorption to the total attenuation (e.g. Jacobson, 1987; Korn, 1990; Fehler et al., 1992). The MultipleLapse Time Window Analysis of Fehler et al. (1992) and Hoshiba (1993) is probably the most popular method in terrestrial seismology and has been applied in a variety of contexts. However, it requires a relatively good epicentral distance coverage, a condition that is not fulfilled on Mars or on the Moon. For this reason, time delay and coda quality factor have remained popular in planetary seismology. BlanchetteGuertin et al. (2012) proposed conceptual models of scattering stratification in the Moon based on measurements of these two parameters. Their work was continued by Gillet et al. (2017) who developed a method based on the diffusion model to retrieve the scattering attenuation profile of the Moon from time delay and $Q_{c}$ measurements. They found that porosity and fractures could extend deeper than the crust-mantle boundary (up to 80 - $100 \mathrm{~km}$ below the surface), as proposed in Lognonné et al. (2003) and in agreement with gravimetric estimates from the GRAIL mission (Zuber et al., 2013).

While mathematically convenient, the diffusion model is only a scalar approximation that describes the transport of the total seismic energy. It relies on the assumption that seismic waves have been scattered so many times that $\mathrm{P}$ and $\mathrm{S}$ polarizations emitted at the source can no longer be distinguished at the detection. The diffusion model predicts simple seismogram envelopes characterized by a rise of the energy to a maximum followed by a monotonous coda decay. While such an approximation is well suited for moonquakes, it cannot be easily reconciled with the observation of two pulses in HF and VF martian events. We will therefore employ an elastic radiative transfer approach in this work. Transport equations for elastic waves have solid theoretical foundations (Weaver, 1990; Ryzhik et al., 1996) and can be conveniently solved by Monte-Carlo simulations (Margerin et al., 2000; Shearer \& Earle, 2004; Przybilla et al., 2009; Gaebler et al., 2015; Sanborn et al., 2017). 
Recently, Lognonné et al. (2020) used the radiative transfer equation to model the full envelope of the first VF event detected by SEIS-InSight (S0128a). They inferred first estimates of crustal diffusivity and intrinsic attenuation indicating that the scattering strength is more Earth-like than Moon-like. The apparent intrinsic attenuation was found to be very low as compared to the Earth, yet slightly higher than on the Moon. van Driel et al. (2021) analyzed tens of HF and VF events and proposed a conceptual model of long range propagation in a waveguide to explain their characteristics. To support their model, they conducted a large number of numerical experiments of wave propagation in a 2-D heterogeneous waveguide and proposed a stratification of heterogeneity in the crust of Mars, with a strongly scattering layer in the first ten kilometers of the planet. In this work, we follow Lognonné et al. (2020) and invert for the scattering and absorption properties of the Martian lithosphere using a radiative transfer approach in a 3-D random elastic half-space. We assume that the fluctuations of elastic parameters can be described (to second order) by a Von Karman correlation function. As shown by Sato (2019), this model provides a simple but realistic mathematical description of heterogeneity on a wide range of spatial scales.

The paper is organized as follows. In section 2 , we introduce the dataset. In section 3, we present analyses of delay time and $Q_{c}$ measurements. For the latter parameter, we compare our results to previous measurements on Earth and on the Moon. In section 4, we present our forward modeling approach of the seismogram envelopes and the inversion strategy. In section 5, we show that the envelopes of VF events may be reasonably well explained by the multiple-scattering of elastic waves in a heterogeneous half-space. The role of the model parameters is analyzed before closing the paper with some conclusions. 


\section{Data description}

The seismometer SEIS is equipped with both a three-axes Very Broad Band (VBB) instrument and a three-axes short-period (SP) instrument (Lognonné et al., 2019). These six sensors cover a broad seismic bandwidth, from $0.01 \mathrm{~Hz}$ to $50 \mathrm{~Hz}$. Throughout the first year of the mission, considerable work was done on the data collected by SEIS to distinguish between seismic and non-seismic signals (Clinton et al., 2021; Ceylan et al., 2021). 465 potential seismic events were identified by the MQS (Clinton et al., 2021; InSight Marsquake Service, 2020) and we have used waveforms provided by the Mars SEIS Data service (Insight Mars SEIS Data Service, 2019a). Based on the spectral content, two families of events can be distinguished: low frequency and high frequency. The low-frequency family includes long period (LF) and broadband (BB) events. Most of the LF energy is at periods greater than 1s. They can be distinguished from (BB) events by the fact that they do not excite the 2.4 $\mathrm{Hz}$ resonances (Clinton et al., 2021). The high-frequency family is composed of socalled "2.4 Hz", high-frequency (HF) and very high-frequency (VF) events. The 2.4 Hz events have narrow spectral content and were interpreted by van Driel et al. (2021) as small HF events. HF are most energetic in the $1-5.5 \mathrm{~Hz}$ frequency band with their spectra decaying rapidly at higher frequencies. VF events have energy up to at least $10 \mathrm{~Hz}$, sometimes even much higher frequencies. The present study focuses on HF and VF events which, thanks to their good signal-to-noise ratio and broad frequency range are most interesting to place constraints on the high-frequency attenuation in Mars.

With the latest seismic event catalog, 75 event waveforms were available for analysis, composed of $52 \mathrm{HF}$ events and $23 \mathrm{VF}$ events (Clinton et al., 2021). Nevertheless, a large number of waveforms are contaminated by non-seismic signals such as donks. Ceylan et al. (2021) defined donks as brief high-frequency events of great intensity, resulting likely from the thermal cracking of the lander. We visually inspected all the waveforms and rejected those that were manifestly too contaminated to be usable for 
coda analysis. Furthermore, we eliminated all the events with a coda duration inferior to $200 \mathrm{~s}$ with a signal-to-noise ratio greater than 2 at $2.5 \mathrm{~Hz}$. After application of these criteria $13 \mathrm{HF}$ and $8 \mathrm{VF}$ waveforms were retained for further analysis. They were collected from the VBB or SP sensors with acquisition frequencies of 20 samples per second (sps) or $100 \mathrm{sps}$, respectively. The arrival times of the primary and secondary arrivals were identified from the envelopes of the signals filtered in different frequency bands. The envelope of the signal is obtained by performing successively the following operations: filtering, squaring and smoothing with a moving average window of $20 \mathrm{~s}$ duration. The events are listed in Table 1.

To give a rapid overview of the high-frequency Martian seismic events, vertical component seismograms and spectrograms of a VF (S0334a) and an HF event (S0314b) are shown in Figure 1. Time windows of $10 \mathrm{~s}$ are used for spectrogram computations and the displacement waveforms are bandpass filtered at $3.5 \mathrm{~Hz}$. Prior to the computations, the instrumental response of the VBB sensor has been removed from the signal. Note that besides the seismometers, SEIS is also composed of a tether connecting the sensor to the lander and a relaxation loop (LSA). The impact of these last two components on the recorded high-frequency ground motion is not perfectly known at the time of writing.

The HF and VF seismograms have an emergent character and a duration that typically exceeds 10 minutes. While both events strongly excite the $2.4 \mathrm{~Hz}$ resonances first reported in Lognonné et al. (2020) and Giardini et al. (2020), the HF event exhibits most of its energy between 1.5 and $5.5 \mathrm{~Hz}$, while the spectral content of the VF ranges from $1.5 \mathrm{~Hz}$ to the Nyquist frequency $(10 \mathrm{~Hz})$. On the horizontal components, this effect is even more pronounced and the displacement spectrum of some of the most energetic VF events is rising above $4 \mathrm{~Hz}$, as illustrated in Figure 2. The origin of the difference between the spectra of VF and HF events is not understood yet. Simple earthquake scaling laws (e.g. Madariaga, 
1976) suggest, for example, that VF events may exhibit larger stress drop than HF events. Nevertheless, such an interpretation requires that all path effects be removed from the spectra shown in Figure 2, an issue which we start to address in this paper.

On each vertical component seismogram, two arrivals can be clearly identified, each followed by a long coda. Unsuccessful attempts at recovering the polarization information of the dominant arrivals suggest that the HF wavefield is almost entirely depolarized. These features are broadly consistent with the observations reported in Clinton et al. (2021). A number of non-seismic signals are also visible in Figure 1. Short, repetitive bursts of high-frequency energy can be discerned, that correspond likely to donks but sometimes also glitches that are associated with high-frequency precursors (Scholz et al., 2020). The permanent narrow band of energy visible at $1 \mathrm{~Hz}$ is caused by cross-talk between the temperature acquisition (at $1 \mathrm{sps}$ ) and the seismic channel (Zweifel et al., submitted; Compaire et al., 2021), termed tick-noise. While the most energetic quality B high-frequency events are of VF type, a large number of HF and VF events have small spectral amplitude and are prone to contamination by environmental and instrumental noise. This is a topic of concern and we shall carefully examine the impact of the noise on our measurements.

\section{Envelope characteristics of $\mathrm{HF}$ and VF events}

\subsection{Analysis of VF event S0734a}

In this section, we present a complete analysis of the VF event S0734a using 20 sps data from the VBB sensor (see also Appendix A for an analysis based on 100 sps SP data). In the range of frequencies where the two datasets overlap, we found good agreement between the results obtained from the VBB and SP sensors. Our objective is to extract the main characteristics of the energy envelopes in different frequency bands. Before performing the measurements, we ap- 
plied the following preliminary processing to the data: detrend using cubic splines, instrumental response correction and finally rotation to North East and Vertical components. In a second step, the energy envelopes are calculated as follows. The data are band-pass filtered, squared and smoothed. We applied Butterworth filters in successive frequency bands $[3 f / 4,5 f / 4]$ centered around frequency $f \in\{2.5,3.5,4.5,5.5$, 6.5, 7.5\} for VBB data (Lognonné et al., 2020) and $f \in[2.5,19.5]$ with a step of 1 $\mathrm{Hz}$ in the case of SP data. We used smoothing windows of 20 seconds to reduce the envelopes fluctuations. We normalized each envelope by dividing the (instantaneous) energy of the signal by the mean energy of the noise computed in a time window of 100 s duration preceding the event start. The results are illustrated in Figure 3.a (for VBB data) and Figure A.1.a (for SP data) where we show the normalized energy envelope of the data in various frequency bands. The square root of these envelopes may readily be interpreted as the signal-to-noise $(S / N)$ ratio of the event. At its maximum, the $S / N$ ratio can be as large as 30 around $8.5 \mathrm{~Hz}$ and is typically above 10 from $2 \mathrm{~Hz}$ to at least $12 \mathrm{~Hz}$. Note that this VF event carries energy almost up to the Nyquist frequency of the SP data $(50 \mathrm{~Hz})$.

Looking at the envelope shape in more details, we observe two wave packets separated in time by approximaltely $80 \mathrm{~s}$, each followed by a pronounced coda. For comparison, we show in Figure 4.a the result of applying the same processing to a regional event recorded on the Canadian shield. This event was chosen on the basis of its similarity with VF S0734a in terms of $\left(t_{s}-t_{p}\right)$ and coda duration. The main phases visible on the terrestrial seismogram are the refracted mantle waves $P_{n}$ and $S_{n}$ as well as the guided $L_{g}$ waves. This last phase, which highlights the velocity discontinuity at the crust/mantle boundary on Earth, distinguishes the anatomy of a regional earthquake from the one of a regional marsquake. The $L_{g}$ wavetrain is followed by a long coda whose decay properties will be analyzed and compared to the ones of VF S0734a. 
In the next subsections we further examine the characteristics of the VF event by analyzing the coda quality factor $Q_{c}$ and delay time $t_{d}$.

\subsubsection{Coda quality factor $\left(Q_{c}\right)$ measurements}

Since the works of Aki \& Chouet (1975), it is widely accepted that the temporal decay of the smoothed energy envelope of the coda may be parameterised as:

$$
E(t) \propto \frac{1}{t^{\alpha}} e^{-\frac{\omega t}{Q_{c}}},
$$

where $Q_{c}$ is the coda quality factor, $\omega$ is the central angular frequency of the signal and $\alpha$ is an exponent that depends on the model geometry. In this work, we will consider both $\alpha=0$ and $\alpha=1$. The former choice facilitates the comparison with lunar measurements presented in Gillet et al. (2017), while the latter value has been traditionally adopted to study the coda decay at regional distances on Earth (Mitchell, 1995). Note that in the case $\alpha=0$, the Aki and Chouet formula is left invariant by a translation of the time origin so that quake location is not needed to measure $Q_{c}$. In the case $\alpha=1$, we determined an approximate origin time $t_{0}$ for the marsquake event. Assuming constant velocities, we have $t_{0}=t_{p}-\left(t_{s}-t_{p}\right) /(\gamma-1)$ where the $V_{p} / V_{s}$ ratio $(\gamma)$ is assumed equal to 1.65 which is realistic for the near surface of Mars. We also tested $\gamma=1.85$, without significant impact on the results.

To measure $Q_{c}$, we perform a robust linear least-squares regression of the logarithm of the envelope of the summed energies of horizontal components, after correction by a factor $t$ ( $t$ is the time elapsed since the event occurence) in the case $\alpha=1$ (Aki \& Chouet, 1975). The start time and end time of the coda windows used for analysis are listed in Table 1. For all the events analyzed, the duration of the coda window is of at least $100 \mathrm{~s}$ duration with $S / N$ greater than 2. The use of robust linear least-squares circum- 
vents the difficulty of performing an accurate linear regression when the signal is contaminated by outliers, in particular donks in the highest frequency range. Furthermore, for events located in the same range of epicentral distance, we took care of selecting very similar time windows in order to minimize possible selection biases. Examination of Figure 3.a on which we have superposed the results of the linear regression with $\alpha=1$ on the data shows that the Aki and Chouet formula approximates the coda decay of marsquakes extremely well. As a consequence, the uncertainties on individual $Q_{c}$ measurements are never larger than 1\%. In Figure 3.a, it is worth noting that seismogram envelopes at different frequencies are almost parallel to one another in the coda. This property is reflected in the almost linear increase of the coda quality factor $Q_{c}$ shown in Figure 3.b (see also Figure A.1.b for SP data). In the Figure, the color bar indicates the average $S / N$ ratio measured on the total energy of horizontal components. To confirm the almost linear trend visible in Figure 3.b, we performed a fit of the frequency dependence of $Q_{c}$ using a parameterisation of the form $Q_{c}=Q_{0} f^{n}$. Based on linear least-squares applied to the logarithm of $Q_{c}$, we obtain $Q_{0}=440 \pm 14$ and $n=1.08 \pm 0.02$ in the $[2.5 \mathrm{~Hz}-7.5 \mathrm{~Hz}]$ range for VBB data with $\alpha=1$ (see Appendix A for the same analysis on SP data with similar results). In the case $\alpha=0$, we find $Q_{0}=422 \pm 13$ and $n=1.07 \pm 0.02$ with an equally satisfactory fit to the data. We note that the choice of $\alpha$ impacts the value of $Q_{0}$ but leaves $n$ practically unchanged. The change in $Q_{0}$ is rather modest, typically less than $10 \%$ for VF S0734a. A visual comparison between Figures 3.a and 4.a reveals a clear difference in the frequency-dependence of the coda decay between Mars and Earth (Canada). In the case of the regional earthquake, the ensemble of decay curves is fanshaped, corresponding to a frequency exponent $n$ lower than 1 . Indeed, a linear regression of $Q_{c}$ measurements shown in Figure $4 \mathrm{~b}$ indicates $Q_{0}=740 \pm$ 74 and $n=0.49 \pm 0.05$. Coda decay properties of earth- and mars-quakes will 
be further compared after analysis of all the selected high frequency events.

\subsubsection{Delay-time $\left(t_{d}\right)$ measurements}

In random heterogeneous media, multiple scattering results in the broadening of energy envelopes with hypocentral distance (Saito et al., 2002; Sato, 1982). This phenomenon may be simply quantified by measuring the rise time from the onset to the peak of a given seismic arrival. To facilitate the comparisons with terrestrial seismology, we define the delay time on the secondary arrival as: $t_{d}=t_{\max }-t_{s}$ where $t_{\max }$ is the arrival time of the energy maximum. In previous studies, delay-time measurements were successfully applied to characterize qualitatively the scattering properties of the medium in both the Earth and the Moon (e.g. Blanchette-Guertin et al., 2012; Gillet et al., 2017; Calvet \& Margerin, 2013). Sophisticated delay time tomography based on multiple-forward scattering approximations have also been developed to map spatial variations of heterogeneity (Takahashi et al., 2007, 2009). The discussion that follows is mostly qualitative and will serve to highlight some differences between Mars, Earth and Moon. Pursuing our analysis of the VF event S0734a, we performed delay-time measurements $\left(t_{d}\right)$ on the seismogram envelopes of the VBB data in the $2.5-7.5 \mathrm{~Hz}$ (see Appendix A.c for an analysis at higher frequencies using SP data). The results are reported in Figures 3.c.

A rather striking feature of the results plotted in Figures 3.c is the absence of clear frequency dependence of the time-delay. This is particularly notable on the VBB data from $2 \mathrm{~Hz}$ to $8 \mathrm{~Hz}$. Time delay of P-waves has received little attention on Earth (see however the work of Kubanza et al., 2007). In the case of the Moon, the diffuse character of the signal allows for the measurement of a single time delay, most likely related to S-waves. For these reasons, the discussion will be focused on the properties of the secondary arrival. On Earth, $t_{d}$ of S-waves has been shown to increase with frequency in various geological contexts (Takahashi et al., 2007; Calvet et al., 
2013). This property is usually understood as a consequence of strong forward scattering and should not be confused with the dispersion of velocities induced by attenuation (whether it is caused by scattering or absorption). For narrowly band-passed waves propagating in a heterogeneous medium, the effect of velocity dispersion on the envelope shape is usually very small compared to the multi-pathing effects induced by forward scattering (Sato, 1989). In the case where the randomness is described by Gaussian spatial correlations of the fluctuations (or more generally smooth random media), Markov's approximation predicts that $t_{d}$ is independent of the central frequency of the waves. The frequency exponent inferred from Earth data is usually strictly positive and sometimes larger than 1 , which is interpreted as the presence of small-scale fluctuations that are not captured by the Gaussian model (Saito et al., 2002; Takahashi et al., 2007). Note that in the case of the Canadian shield event, the level of scattering attenuation is presumably very low. In this example, the dominant mechanism at the origin of the peak delay is most likely the multiple reverberations of the guided waves in the crust.

In sharp contrast with the Earth, $t_{d}$ is seen to decrease with frequency on the Moon (Gillet et al., 2017). There is no consensus explanation for this fact. But it is important to keep in mind that $t_{d}$ is sensitive to both scattering and attenuation and the latter mechanism decreases the time delay (see Sato, 1989). Attenuation does add some dispersion to the seismic velocities but since we are considering multiplescattering of narrow-band signals, the effect is tiny. Mars displays yet another behavior, somehow intermediate between Earth and Moon with apparently little frequency dependence of $t_{d}$ for the second arrival. As a consequence, for each event we have estimated the mean value of $t_{d}$ and its uncertainty based on a set of measurements on filtered data in the $[3 f / 4,5 f / 4]$ band, where the central frequency increases from $2.5 \mathrm{~Hz}$ to $19.5 \mathrm{~Hz}$ by steps of $1 \mathrm{~Hz}$. In Figure 5 , we report our measurements of $t_{d}$ as a function of the time difference $\left(t_{s}-t_{p}\right)$ between the second and first arrivals, which 
can be understood as a proxy for the hypocentral distance $R$. While multiple-forward scattering theory predicts that $t_{d}$ increases like $R^{2}$ in smooth random media (Saito et al., 2002), the delay time of high-frequency martian events appears to be remarkably independent on $\left(t_{s}-t_{p}\right)$. This property distinguishes Mars from Earth and Moon where the increase of $t_{d}$ with hypocentral distance is clearly observed (Takahashi et al., 2007; Blanchette-Guertin et al., 2012). The deviation of martian HF events from the predicted theoretical behavior can have different origins: competition between scattering and attenuation (absorption), contribution of large angle scattering neglected in Markov's approximation or non-uniformity of scattering properties. These hypotheses will be evaluated in the light of envelope modeling in section 4 .

\subsection{Comparison of $\mathrm{HF}$ and $\mathrm{VF}$ events characteristics}

The preliminary analysis of HF and VF events revealed significant differences in spectral content (see Figures 1-2). In this section, we propose to extend the comparison between the two groups of events by examining the coda quality factor and delay time of $13 \mathrm{HF}$ and $8 \mathrm{VF}$ events in the $2.5-5.5 \mathrm{~Hz}$ and $2.5-7.5 \mathrm{~Hz}$ frequency bands, respectively. We only use data collected on the VBB sensors with sampling rates of 20 sps.

\subsubsection{Delay time}

Similar to the VF event S0734a (Figure 3), the delay-time $\left(t_{d}\right)$ measured for each of the HF and VF events appears to vary little with frequency. The apparent differences in the measurements of $t_{d}$ at different frequencies are likely the results of statistical fluctuations of the envelopes or contamination of the signal by donks. As a consequence, we define for each event the delay-time and its uncertainty as the mean and the standard deviation to one sigma of all the values measured on the filtered data in the $[3 f / 4,5 f / 4]$ band with $f \in\{2.5,3.5, \cdots, 7.5\}$. Figure 5 (left) reports the delay-time as a function of $t_{s}-t_{p}$, taken as a proxy for the hypocentral distance. On 
this Figure, there is no clear-cut difference between the VF and HF events, except that the latter show more variability. The lack of information on the back-azimuth of these events makes it impossible to determine whether this variability reflects a genuine path effect or if this is just a consequence of noise contamination. Indeed, the small amplitude of the HF events that we have analyzed makes them more sensitive to the noise conditions, as will be illustrated later.

\subsubsection{Coda quality factor}

Figure 5 (right) shows the dependence of the coda quality factor of HF and VF events at $3.5 \mathrm{~Hz}$ as a function of $\left(t_{s}-t_{p}\right)$. Dark and light colors correspond respectively to the choice $\alpha=0$ and $\alpha=1$ in formula (1). We observe again a much greater variability of $Q_{c}$ among $\mathrm{HF}$ events. This observation has to be interpreted with some caution. While it suggests that some HF events sample a significantly higher $Q$ region of Mars as compared to VF events, we will show in section 3.3 that the coda decay is extremely sensitive to the noise conditions. This is all the more true as the $Q$ values (typically in the range $2000-9000$ at $3.5 \mathrm{~Hz}$ ) are particularly high. If we make abstraction of HF events, there appears to be an increase of the coda quality factor with hypocentral distance. Since the more distant events are likely to sample deeper parts of the medium as compared to the nearest one, this increase is suggestive of a possible stratification of attenuation properties with depth. This hypothesis will be further explored in section 5 .

In preparation of section 3.3, we continue our exploration of HF and VF events with the analysis of Figure 6 which reports our measurements of $Q_{c}$ as a function of the signal-to-noise ratio for central frequencies $f \in\{2.5,3.5, \cdots, 7.5\} \mathrm{Hz} . S / N$ and its uncertainty were evaluated in a $200 \mathrm{~s}$ long window starting at the onset of the second arrival. As a consequence of their narrower spectral content, the coda quality factor of $\mathrm{HF}$ events could only be measured in the two lowest frequency bands $(2.5-3.5 \mathrm{~Hz})$. The difference between the two groups of events is apparent on both axes of Figure 6: 
HF events show on average higher $Q_{c}$ and lower $S / N$ than VF events. The variability of $Q_{c}$ for $\mathrm{HF}$ events at $3.5 \mathrm{~Hz}$ (Figure 5) is thus largely confirmed at $2.5 \mathrm{~Hz}$. While this observation suggests that HF events sample on average higher Q regions of Mars, it is important to keep in mind the influence of the noise on the measurements. This point is further explored in the next subsection.

\subsection{Noise sensitivity analysis}

To understand the impact of the noise on envelope characteristics, we performed a simple sensitivity analysis by adding noise data to one the VF events (S0334a). We selected a noise window during Sol 333 at the exact local time of occurrence of S0334a. Before addition to the event signal, the noise amplitude was scaled by a factor $k \in\{0,1,2,3,4,5\}$. Figure 7 shows the results of the noise addition to the signal of S0334a from $2.5 \mathrm{~Hz}$ to $7.5 \mathrm{~Hz}$. As hypothesized above, the impact of the noise level on the coda quality factor is quite large. As the $S / N$ ratio decreases by a factor 4 , the quality factor increases by a factor almost 3 at $3.5 \mathrm{~Hz}$. This very high sensitivity of $Q_{c}$ to the noise level has two origins: (1) the $S / N$ of the original signal is not very high (of the order of 10) and (2) The rate of decay of the coda is rather slow. By "slow", we mean that the coda length is not very large compared to the typical decay time $Q_{c} / \omega$ at circular frequency $\omega$. Hence, a small amount of noise can lead to an overestimation of $Q_{c}$. Comparing Figures 6 and 7, we recognize a somewhat similar pattern, suggesting that part of the variability of $Q_{c}$ among $\mathrm{HF}$ events may be explained by their low $S / N$ ratio. Finally, we remark that the impact of the noise on the coda decay of VF events at higher frequency $(7.5 \mathrm{~Hz})$, while slightly less pronounced than at $3.5 \mathrm{~Hz}$, is still noticeable.

\subsection{Coda attenuation: Earth-Moon-Mars comparison}

To illustrate the variability of the coda decay on Mars, we show in Figure 8 the values of $Q_{0}$ and $n$ for $8 \mathrm{VF}$ events analyzed in this study. HF are 
not shown as their bandwidth is too narrow for meaningful comparison with Earth. Figure 8 confirms that the value of $\alpha$ mostly impacts $Q_{0}$, but not $n$. Like in the case of VF S0734a, we find that the value of $Q_{0}$ typically increases by no more than $10 \%$ when substituting $\alpha=0$ with $\alpha=1$ in formula (1). To summarize our results, we perform an average over all events and find $Q_{0}=$ $701 \pm 188$ and $n=1.04 \pm 0.1$ for $\alpha=1\left(Q_{0}=644 \pm 156\right.$ and $n=1.038$ for $\left.\alpha=0\right)$. The measurements with $\alpha=1$ (resp. $\alpha=0$ ) will be compared to $Q_{c}$ values for Earth (resp. Moon). We remind the reader that $\alpha=1$ is commonly used in coda attenuation studies at regional distances (Mitchell, 1995), while $\alpha=$ 0 has been adopted in the case of the Moon (Blanchette-Guertin et al., 2012; Gillet et al., 2017). In Figure 9, we have compiled a number of $Q_{c}$ measurements performed in different geological environments on Earth together with data from the Moon and the results obtained in this study for Mars. Before discussing the Figure in more details, we note that while studies performed at regional distances on Earth make use of $\alpha=1$ (e.g. Xie \& Mitchell, 1990; Mitchell et al., 2008), a number local coda $Q$ studies, particularly in tectonic areas on Earth, employ $\alpha=2$. The latter value differs from the one used in this study. Since raising $\alpha$ by one unit increases $Q_{0}$ by less than $10 \%$ and does not affect $n$ (for martian data), we believe that the comparison is nevertheless meaningful.

With $Q_{0} \approx 700$, the coda quality factor on Mars lies in the upper range of the values observed on Earth, particularly when compared to measurements reported for tectonically active regions (in blue in Figure 9) where $Q_{0}$ typically varies from 100 to 400 (see Table 2 for references). It is only in cratons or shields (shown in green and yellow in Figure 9) that $Q_{0}$ exceeds 600 and can in certain regions be as high as 1000 (Singh \& Herrmann, 1983; C. R. Woodgold, 1990; Xie \& Mitchell, 1990; Singh et al., 2004; Mitchell et al., 2008). Figure 9 clearly shows that the frequency dependence of $Q_{c}$ on Earth is radically 
different between tectonic areas and shields, with an approximately linear increase of $Q_{c}$ for the former $(n \approx 1)$, and a frequency exponent in the range 0.1-0.5 for the latter (see also Romanowicz \& Michell, 2015, for a review). In fact, there appears to be a systematic correlation between $Q_{0}$ and $n$ on Earth, with high $Q_{0}$ generally associated with small $n$. This is for instance particularly obvious on the Lg coda Q tomographies by Xie \& Mitchell (1990) for Africa (see a number of other examples in Romanowicz \& Michell, 2015). Furthermore, $Q_{c}$ appears to saturate at high-frequency on Earth and only exceptionally exceeds 3000 at $20 \mathrm{~Hz}$ (e.g. C. R. Woodgold, 1990; C. Woodgold, 1994). No such saturation is seen in the VF events data.

On the Moon, Gillet et al. (2017) report coda $Q_{c}$ values of 2400 at $0.5 \mathrm{~Hz}$ and possibly as large as 10000 at $7 \mathrm{~Hz}$. From these observations, it appears that $Q_{0}$ on the Moon must be a factor 2 to 4 larger than on Mars, as already suggested in Lognonné et al. (2020). The frequency dependence is less well constrained due to the limited bandwidth of the seismic sensors deployed in the framework of the Apollo mission. Nevertheless, the values reported above suggest that the frequency exponent for $Q_{c}$ on the Moon lies more likely in the range $0.4-0.6$.

In summary, we see that $Q_{c}$ on Mars is lower than on the Moon (at the same frequency) but increases more rapidly with frequency. The unique combination of high $Q_{0}$ with a frequency exponent $n \approx 1$ also distinguishes very clearly Mars from the Earth. The quasi-linear frequency increase of $Q_{c}$ seen on VF events suggests that the coda decay is governed by a frequency independent attenuation time.

In the next section, we will focus on the highest quality VF events and infer the average scattering and absorption properties of the martian lithosphere from the multiple-scattering modeling of their envelope shape. 


\section{Seismogram envelopes modeling}

In this section, we first present the theoretical approach and the inversion procedure to retrieve the scattering and attenuation properties of Mars from the modeling of seismogram envelopes of VF events. The method is illustrated on three VF events covering a broad range of hypocentral distances.

\subsection{Radiative transfer model.}

The envelope characteristics and the statistical properties of the signal of HF Martian events suggest that they are composed of seismic waves that have propagated in the multiple scattering regime. To describe the spatio-temporal distribution of seismic energy and the energy exchange between different modes of propagation, we use an approach based on the elastic radiative transport model in a half-space (Turner \& Weaver, 1994; Ryzhik et al., 1996). We suppose that the first and second energy 'packets' observed during VF events correspond to multiply-scattered P and S waves emitted by a single impulsive source. To model the energy envelopes, we use the MonteCarlo method to solve the following simplified set of transport equations (Margerin, 2017):

$$
\begin{aligned}
\left(\partial_{t}+c^{p} \mathbf{n} \cdot \nabla\right) \rho^{p}(\mathbf{r}, \mathbf{n}, t)= & -\left(\frac{1}{\tau^{p}}+\frac{1}{t_{i}^{p}}\right) \rho^{p}(\mathbf{r}, \mathbf{n}, t)+\frac{1}{\tau^{p p}} \int_{4 \pi} p^{p p}\left(\mathbf{n}, \mathbf{n}^{\prime}\right) \rho^{p}\left(\mathbf{r}, \mathbf{n}^{\prime}, t\right) d^{2} \mathbf{n}^{\prime} \\
& +\frac{1}{\tau^{s p}} \int_{4 \pi} p^{s p}\left(\mathbf{n}, \mathbf{n}^{\prime}\right) \rho^{s}\left(\mathbf{r}, \mathbf{n}^{\prime}, t\right) d^{2} \mathbf{n}^{\prime}+S^{p}(\mathbf{r}, \mathbf{n}, t) \\
\left(\partial_{t}+c^{s} \mathbf{n} \cdot \nabla\right) \rho^{s}(\mathbf{r}, \mathbf{n}, t)= & -\left(\frac{1}{\tau^{s}}+\frac{1}{t_{i}^{s}}\right) \rho^{s}(\mathbf{r}, \mathbf{n}, t)+\frac{1}{\tau^{p s}} \int_{4 \pi} p^{p s}\left(\mathbf{n}, \mathbf{n}^{\prime}\right) \rho^{p}\left(\mathbf{r}, \mathbf{n}^{\prime}, t\right) d^{2} \mathbf{n}^{\prime} \\
& +\frac{1}{\tau^{s s}} \int_{4 \pi} p^{s s}\left(\mathbf{n}, \mathbf{n}^{\prime}\right) \rho^{s}\left(\mathbf{r}, \mathbf{n}^{\prime}, t\right) d^{2} \mathbf{n}^{\prime}+S^{s}(\mathbf{r}, \mathbf{n}, t)
\end{aligned}
$$

where $\tau^{\alpha \beta}$ is the $\alpha$ to $\beta$ scattering mean free time $(\alpha, \beta \in\{P, S\})$. In addition, we have introduced the following notations: $t_{i}^{\alpha}$ and $c^{\alpha}$ are the absorption time and the velocity of the mode $\alpha, \rho^{\alpha}(\mathbf{r}, \mathbf{n}, t)$ is the energy density propagating in direction $\mathbf{n}$ at time $t$ and position $\mathbf{r}$ of mode $\alpha . S^{\alpha}(\mathbf{r}, \mathbf{n}, t)$ is the source term for mode $\alpha$. The local angular 
scattering pattern $p^{\alpha \beta}\left(\mathbf{n}, \mathbf{n}^{\prime}\right)$ is the probability that $\alpha$ energy propagating in direction, $\mathbf{n}^{\prime}$ is converted into $\beta$ energy propagating in direction $\mathbf{n}$. Finally, $1 / \tau^{\alpha}=\sum_{\beta} 1 / \tau^{\alpha \beta}$ is the total inverse scattering mean free time (of mode $\alpha$ ). Note that the set of Eq. (2) can be reduced to a simple scalar diffusion equation for the total energy density $\left(\rho^{p}+\rho^{s}\right)$ in the limit of long propagation times $(t \rightarrow \infty)$. To facilitate the comparison between Mars and the Moon, we will therefore quantify the strength of the scattering by computing the equivalent seismic diffusivity of the models of heterogeneity derived from the inversion of seismic envelopes. For a presentation of the formula for the diffusivity in a seismological context, the reader is referred to Turner (1998). To facilitate the comparison with the Earth, we will also quantify the scattering strength with the aid of the shear wave quality factor $Q_{s c}=\omega \tau^{s}$, where $\omega$ is the central frequency of the waves.

As demonstrated by scattering theory, the angular patterns $p^{\alpha \beta}$ are governed by the power spectrum of the random fluctuations as well as the possible correlations among the elastic parameters (Sato et al., 2012). In this work, we make the classical assumption that density and velocity are correlated as follows: $\delta c^{p} / c^{p}=\delta c^{s} / c^{s}$ and $\delta \rho / \rho=\nu \delta c^{p} / c^{p}$. This is known as Birch law in the literature. In this work, we adopt the classical value $\nu=0.8$ for lithospheric rocks (Sato, 1984). The power spectrum of the elastic fluctuations $\Phi$ is assumed to be of Von-Karman type:

$$
\Phi(m)=\frac{(2 \sqrt{\pi})^{3} \Gamma(\kappa+3 / 2)\left\langle\epsilon^{2}\right\rangle l_{c}^{3}}{\Gamma(\kappa)\left(1+m^{2} l_{c}^{2}\right)^{\kappa+3 / 2}}
$$

where $m$ is the wavenumber (of the fluctuations), $\Gamma$ is the Gamma function, and $\left\langle\epsilon^{2}\right\rangle$ is the variance of the fluctuations. The two key parameters in Eq. (3) are the Hurst exponent $\kappa$ and the correlation length $l_{c}$. The exponent $\kappa$ controls the richness of the medium in small-scale fluctuations and $l_{c}$ is the typical size of the largest heterogeneities. A classical choice for $\kappa$ is 0.5 , corresponding to a correlation function of 
exponential type. In this work, we will explore other forms of correlation corresponding to smoother media $(\kappa=2)$ besides the usual case $\kappa=0.5$.

To complete the presentation of the model we discuss briefly the source term, absorption model and average wavespeeds. In view of the broad similarity of VF events with high-frequency earthquakes, it is reasonable to assume that they are of internal origin. In our model, the $P / S$ energy ratio emitted at the source is therefore calculated from the radiation of a double couple and depends on the velocity ratio $c^{p} / c^{s}$ to the fifth power (Sato et al., 2012). For lack of more precise information, we will assume that the dissipation of seismic energy is caused by shearing motions and is therefore entirely controlled by the rigidity. We therefore have the following relation: $t_{i}^{p}=\frac{3}{4}\left(\frac{c^{p}}{c^{s}}\right)^{2} t_{i}^{s}$, between the absorption times of $\mathrm{P}$ and $\mathrm{S}$ waves. As the seismic wavespeeds are not very well constrained except for the near surface (Lognonné et al., 2020), we will assume that $c^{s}=1.7 \mathrm{~km} / \mathrm{s}$, characteristic of the upper crust, and explore two possible values of the $c^{p} / c^{s}$ ratio $(1.65,1.85)$. We also investigated a higher shear wave speed $c^{s}=3.0 \mathrm{~km} / \mathrm{s}$ and obtained similar fits to the data, with somewhat lower scattering attenuation. We will come back to the role of $c_{s}$ later in the discussion.

\subsection{Inversion procedure}

On Earth, the most common approach to retrieve the scattering and attenuation properties from seismogram envelopes is the Multiple-Lapse-Time-Window-Analysis (Fehler et al., 1992), where the smoothed spatio-temporal distribution of seismic energy is estimated from energy integrals for an ensemble of earthquakes in the vicinity of the station. This method cannot be readily transposed to Mars, due to the lack of seismicity at short hypocentral distance. In the case of the Moon, Gillet et al. (2017) developed an inversion method which uses only $t_{d}$ and $Q_{c}$ to characterize the shape of seismogram envelopes. Again, this approach is not readily applied to our data since VF events are composed of two major energy arrivals. We therefore decided to perform 
a direct inversion of the full seismogram envelope of horizontal components using the transport equations (2) for the forward model.

The inversion seeks to retrieve only two parameters: the scattering mean free time $\left(\tau^{s}\right)$ and the absorption time of $\mathrm{S}$ waves $\left(t_{i}^{s}\right)$. It is very important to note that all the scattering parameters of Eq. (2), i.e. $\tau^{\alpha \beta}$ and $p^{\alpha \beta}$ are entirely determined by $\tau^{s}$ once $\omega$ (central circular frequency), $l_{c}$ (correlation length) and $\kappa$ (Hurst exponent) are fixed. Because the envelope shape does not vary much between 2.5 and $8.5 \mathrm{~Hz}$, we decided to perform the inversion at $6 \mathrm{~Hz}$ to optimize the $S / N$ ratio. Since the correlation length $l_{c}$ is unknown, we explored a broad range of values ranging from a few tens of meters to a few kilometers. For the Hurst exponent, two possibilities were considered: $\kappa=0.5$ (exponential medium) and $\kappa=2.0$ (smooth Von-Karman medium). The best results were obtained with the later choice as illustrated below.

To quantify the agreement between data and model, we apply a simple leastsquares criterion to the difference of the logarithms of the observed and modeled energy envelopes:

$$
\chi^{2}=\int_{0}^{t_{e}}\left[\log \left(\frac{E^{o}(t)}{N^{o}}\right)-\log \left(\frac{E^{m}(t)}{N^{m}}\right)\right]^{2} d t
$$

where $E$ denotes the energy and the integral is computed from the signal onset to the return to the noise level at time $t_{e}$. The use of the logarithm in the definition of $\chi^{2}$ is important to give similar weights to the two main arrivals and the coda in the inversion process. In Eq. (4), the symbols $N^{o, m}$ denote energy integrals that are used to cross-normalize the synthetic and observed envelopes, where the subscripts $o, m$ refer to observations and model, respectively. This is the same idea as the coda normalization method (Aki \& Chouet, 1975; Rautian \& Khalturin, 1978), where source and site effects are removed by normalizing the amplitude of the signal by the energy level of the coda at long lapse-time. In our applications, we used a window of $50 \mathrm{~s}$ durations at the end of the coda (i.e. between $t_{e}-50 \mathrm{~s}$ and $t_{e}$ ). 
To minimize $\chi^{2}$, we employ an iterative optimization approach based on the Levenberg-Marquardt algorithm as implemented in the GNU Scientific Library (Galassi et al., 2017). To guide the inversion process, the method requires the computation of the sensitivity of the synthetic envelope to a perturbation in the model parameters. Rather than the scattering and absorption times, we employ the attenuations $1 / \tau^{s}$ and $1 / t_{i}^{s}$ for the parametrization, as is naturally suggested by the form of Eq. (2). Therefore, in addition to solving Eq. (2), we use the differential Monte-Carlo method of Takeuchi (2016) to compute the partial derivatives of the energy envelopes with respect to the scattering and absorption attenuations. In general, a few iterations suffice to converge to the minimum. The algorithm also provides uncertainties on the best-fitting parameters based on the assumptions that the errors are Gaussian.

\section{Inversion Results}

Some representative results of the inversion procedure are shown in Figure 10 for the VF events S0734a (top), S0756a (middle) and S0421a (bottom). Assuming a uniform shear velocity $c^{s}=1.7 \mathrm{~km} / \mathrm{s}$ and $c^{p} / c^{s}=1.85$ (resp. 1.65), the time difference $\left(t_{s}-t_{p}\right)$ indicates that these events are approximately located at $330 \mathrm{~km}$ (resp. $390 \mathrm{~km}), 670 \mathrm{~km}$ (resp. $780 \mathrm{~km})$ and $1510 \mathrm{~km}$ (resp. $1760 \mathrm{~km})$ from the station, respectively. The agreement between the best-fitting radiative transfer models with Hurst exponent $\kappa=2$ and the data is rather satisfactory, particularly for the two shortest hypocentral distances. It is worth noting that the amplitude ratio between the two dominant arrivals is consistent with the hypotheses that shear dislocations are at the origin of the VF events, and that all dissipation is due to shear. For the most distant event, the quality of the fit degrades. We see in particular that the rise time of the first arrival is too short and its coda decay too rapid. We will further comment on this discrepancy below. In all the cases that we examined, the adimensional correlation length $\omega l_{c} / c^{s}$ is larger than 1 , which indicates that the scattering is predominantly in the forward direction (see eg. Sato et al., 2012, for details). The most important 
preliminary conclusion to be drawn from Figure 10 is that the numerical modeling supports a multiple-forward-scattering origin for the observed VF events waveforms (and most likely other high-frequency events). We will now discuss more precisely the outcome of the inversions by analyzing the sensitivity of our results to the $c^{p} / c^{s}$ ratio and the Hurst exponent. Finally, we will argue that the inversion results suggest a possible stratification of attenuation properties in Mars.

\subsection{Role of the Hurst exponent}

In Figure 10, we compare the outcome of the inversions for the two Hurst exponents: $\kappa=0.5,2.0$. For all the events that we investigated, we observed that the largest value of $\kappa$ systematically yields the best fit to the data. This suggests that Earth is richer in small-scale heterogeneities than Mars. In more informal terms, we may say that the interior of Mars appears smooth as compared to the Earth. It is worth noting that the correlation length derived from the models decreases strongly as $\kappa$ increases. This is an example of trade-off that needs to be resolved in future works. However, we would like to point out that there are indications in the data that $\kappa$ could indeed be larger on Mars than on Earth. We have already noted that the envelope shape of the VF events is remarkably independent of the frequency. In a medium with uniform scattering properties, such a behavior can only be reproduced in the forward-scattering regime $\left(\omega l_{c} / c^{s}>1\right)$ for sufficiently large values of $\kappa$, typically of the order of 2 or larger (see Calvet \& Margerin, 2013). The fact that the best fits are obtained for $\kappa=2$ is therefore consistent with the overall characteristics of the VF seismogram envelopes across multiple frequencies.

\subsection{Impact of the $c^{p} / c^{s}$ ratio.}

The ratio $c^{p} / c^{s}$ in the interior of Mars is so far not very well constrained and it is important to examine how it may affect the fit to the data. The presence of porosity in the shallow part of the crust may reduce $c^{p} / c^{s}$ of the host rock. In Figure 
11 , we examine the case $c^{p} / c^{s}=1.65$ and compare it with the reference models with $c^{p} / c^{s}=1.85$. The Hurst exponent is set to 2 . Overall, the best-fitting models obtained in the two cases are very similar and agree reasonably well with the data, except for the largest hypocentral distance. This result confirms the consistence of the data with a multiple-scattering hypothesis. Reciprocally, we may argue that it is unfortunately not possible to put constraints on the $c^{p} / c^{s}$ ratio in the crust from envelope shapes alone.

\subsection{Variability of scattering and attenuation parameters}

To give an idea of the variability of the scattering and attenuation properties inferred from the envelope shape of VF events, the inversion procedure was applied to seven of them (S0128a, S0264e, S0334a, S0421a, S0424c, S0734a, S0756a) at a central frequency of $6 \mathrm{~Hz}$ with $c^{p} / c^{s}=1.85$ and $\kappa=2$. Figure 12 compiles the results of the inversion for the diffusivity (left), the scattering quality factor of S waves (center) and the intrinsic quality factor of $\mathrm{S}$ waves (right). We see that the diffusivity ranges from 50 to $800 \mathrm{~km}^{2} / \mathrm{s}$, which suggests that the scattering attenuation on Mars is much more Earth-like than Moon-like. For the smallest hypocentral distances, the results are compatible with previous findings by Lognonné et al. (2020), based on the analysis of S0128a. Note that changing the average shear wavespeed to $c_{s}=3 \mathrm{~km} / \mathrm{s}$ increases the inferred diffusivity by a factor approximately equal to 2.8 . This result is a direct consequence of the linear increase of the hypocentral distance with $c_{s}$. By a simple similarity argument based on the diffusion model, the increase of the diffusivity due to an increase of $c_{s}$ is expected to be equal to the ratio of the shear wavespeeds squared $(3 / 1.7)^{2} \approx 3.1$, which is not far from the value 2.8 reported above. As for the attenuation, with an intrinsic quality factor $Q_{\mu}$ ranging from 3500 to 10000, Mars is found to be much more Moon-like than Earth-like. The albedo of the martian lithosphere is extremely high, typically of the order of 0.9 , in sharp contrast with the Earth where the albedo rarely exceeds 0.5 . Some notable exceptions are volcanic areas (e.g. Mayeda et al., 
1992 ) but only at sufficiently low frequencies, typically less than $3 \mathrm{~Hz}$. Recently, Scheller et al. (2021) proposed that vast amounts of water could be trapped in the crust of Mars. This model may be compatible with our observations, provided that some parts of the crust remain mostly dry. That the upper crust could be dry in the equatorial region near InSight is in agreement with the study of Clifford et al. (2010). To put our measurements of $Q_{\mu}$ in perspective, we recall that experiments on basalt samples by Tittmann et al. (1980) have revealed that high $Q$ values (typically higher than 2500) can only be achieved through the complete degassing of the material, including the removal of intra-crystalline water. The $Q_{\mu}$ values we report in the present work are in fact higher than the highest values obtained by Tittman (1977) after several degassing cycles in vacuum. Unless our understanding of the role of water in the attenuation of seismic waves is incorrect, an important conclusion of our study is that the waveforms of VF events are compatible with a mostly dry crust.

In Figure 12, the increase of $D, Q_{s c}$ and $Q_{\mu}$ with hypocentral distance (or equivalently with the proxy $\left.\left(t_{s}-t_{p}\right)\right)$ is particularly clear. In Linear-Log scale, the dependence is approximately linear, which suggests an empirical dependence of the form $y=y_{0} \exp \left(\left(t_{s}-t_{p}\right) / \xi\right)$ where $y$ stands for $D, Q_{s c}$ or $Q_{\mu}$. Indeed, this simple form is seen to fit the observations reasonably well. These results show clearly that, while it reasonably fits the observed seismogram envelopes individually, a uniform scattering model cannot explain simultaneously all the records. Furthermore, we observe that as the hypocentral distance increases, attenuation by scattering and absorption appear to decrease. Under the reasonable assumption that the sensitivity to deep structures increases with hypocentral distance, a natural explanation for the trend seen in Figure 12 is that there exists a stratification of attenuation properties inside Mars. More precisely, we may expect a rapid decrease of attenuation with depth. Recent results by Knapmeyer-Endrun et al. (2021) indicate that, while this is not apparent from the seismogram envelopes of VF events, the crust of Mars is stratified. Determining the depth dependence of Mar- 
tian attenuation taking into account the stratification of velocities in the lithosphere will undoubtedly be an important topic for future works.

\section{Conclusions}

In this paper, we have investigated the envelope and attenuation characteristics of high-quality high-frequency martian events recorded by the instrument SEIS in the framework of the InSight mission. The main conclusion of our investigations is that these events are compatible with the propagation of elastic waves radiated by internal sources in a multiple-scattering medium. This statement is attested by the overall good agreement between synthetic envelopes predicted by elastic radiative transfer models and observed envelopes. Data analysis and modeling has also revealed some outstanding features. (1) As compared to high-frequency quakes observed on Earth or on the Moon, the envelope shape of high-frequency martian events is remarkably weakly dependent on frequency. The coda decay is characterized by an almost constant attenuation, reflected in the linear increase of the coda quality factor with frequency. The delay-time is also very weakly frequency-dependent, in sharp contrast what is observed on earthquakes and moonquakes waveforms. (2) Numerical inversions of the seismogram envelopes of high-frequency martian events with an elastic radiative transfer model show that the observations can be explained by multiple-scattering in smooth models of heterogeneity with a correlation distance in the range 70 - $240 \mathrm{~m}$ and a Hurst exponent $\kappa=2$. The overall strength of the scattering is found to be Earth-like. We also found that the attenuation parameters apparently increase with hypocentral distance, which we interpret as a possible signature of a stratification of heterogeneity in Mars (van Driel et al., 2021). (3) Finally, with a frequency-independent albedo of about 0.9 , we found that attenuation (dissipation of seismic energy) is very low in Mars. This finding indicates that the high-frequency Martian events sample mostly dry materials. This issue requires further investigation. In future works, we propose 
in particular to develop stratified models of scattering and attenuation to test the compatibility of our data with the presence of layers with high levels of dissipation.

\section{Data and Resources}

Seismic data used for this study were collected as part of the NASA InSight (Interior Exploration using Seismic Investigations, Geodesy and Heat Transport, Banerdt et al., 2020) Mission to Mars using SEIS (Seismic Experiment for Interior Structure) seismometer (Lognonné et al., 2019). They can be uploaded on the IRIS Data Management Center website (https://www.iris.edu/hq/sis/insight), the NASA PDS Geoscience Node (InSight SEIS Science Team, 2019) and the IPGP SEIS Data portal (InSight Mars SEIS Data Service., 2019).

\section{Acknowledgments}

We acknowledge NASA, CNES, their partner agencies and Institutions (UKSA, SSO, DLR, JPL, IPGP-CNRS, ETHZ, IC, MPS-MPG) and the flight operations team at JPL, SISMOC, MSDS, IRIS-DMC and PDS for providing SEED SEIS data. This is InSight contribution 183

\section{References}

Aki, K., \& Chouet, B. (1975). Origin of coda waves, sources and attenuation. Journal of Geophysical Research, 80, 3322-3342.

Banerdt, W. B., Smrekar, S. E., Banfield, D., Giardini, D., Golombek, M., Johnson, C. L., , others (2020). Initial results from the insight mission on mars. Nature Geoscience, 13(3), 183-189.

Bianco, F., Del Pezzo, E., Castellano, M., Ibanez, J., \& Di Luccio, F. (2002). Separation of intrinsic and scattering seismic attenuation in the southern apennine zone, italy. Geophysical Journal International, 150(1), 10-22. 
Biswas, K., Mandal, P., \& Khan, P. K. (2019). Estimation of coda q for the eastern indian craton. Journal of Earth System Science, 128(4), 1-16.

Blanchette-Guertin, J.-F., Johnson, C., \& Lawrence, J. (2012). Investigation of scattering in lunar seismic coda. Journal of Geophysical Research: Planets, 117(E6).

Calvet, M., \& Margerin, L. (2013). Lapse-time dependence of coda q: Anisotropic multiple-scattering models and application to the pyrenees. Bulletin of the Seismological Society of America, 103(3), 1993-2010.

Calvet, M., Sylvander, M., Margerin, L., \& Villaseñor, A. (2013). Spatial variations of seismic attenuation and heterogeneity in the pyrenees: Coda $q$ and peak delay time analysis. Tectonophysics, 608, 428-439.

Ceylan, S., Clinton, J. F., Giardini, D., Böse, M., Charalambous, C., van Driel, M., ... others (2021). Companion guide to the marsquake catalog from insight, sols 0-478: Data content and non-seismic events. Physics of the Earth and Planetary Interiors, 310, 106597.

Clifford, S. M., Lasue, J., Heggy, E., Boisson, J., McGovern, P., \& Max, M. D.

(2010). Depth of the martian cryosphere: Revised estimates and implications for the existence and detection of subpermafrost groundwater. Journal of Geophysical Research: Planets, 115(E7).

Clinton, J. F., Ceylan, S., van Driel, M., Giardini, D., Stähler, S. C., Böse, M., ... others (2021). The marsquake catalogue from insight, sols 0-478. Physics of the Earth and Planetary Interiors, 310, 106595.

Compaire, N., Margerin, L., Garcia, R. F., Pinot, B., Calvet, M., Orhand-Mainsant, G., ... others (2021). Autocorrelation of the ground vibrations recorded by the seis-insight seismometer on mars. Journal of Geophysical Research: Planets, e2020JE006498.

De Souza, J., \& Mitchell, B. (1998). Lg coda q variations across south america and their relation to crustal evolution. pure and applied geophysics, 153(2), 587-612. 
Fehler, M., Hoshiba, M., Sato, H., \& Obara, K. (1992). Separation of scattering and intrinsic attenuation for the Kanto-Tokai region, Japan, using measurements of S-wave energy versus hypocentral distance. Geophysical Journal International, 108, 787-800.

Gaebler, P. J., Sens-Schönfelder, C., \& Korn, M. (2015). The influence of crustal scattering on translational and rotational motions in regional and teleseismic coda waves. Geophysical Journal International, $201(1)$, 355-371.

Galassi, M., Davies, J., Theiler, J., Gough, B., Jungman, G., Alken, P., ... Ulerich, R. (2017). GNU Scientific Library Reference Manual.

Giampiccolo, E., Gresta, S., \& Rascona, F. (2004). Intrinsic and scattering attenuation from observed seismic codas in southeastern sicily (italy). Physics of the Earth and Planetary Interiors, 145(1-4), 55-66.

Giardini, D., Lognonné, P., Banerdt, W. B., Pike, W. T., Christensen, U., Ceylan, S., . . others (2020). The seismicity of mars. Nature Geoscience, 13(3), 205-212.

Gillet, K., Margerin, L., Calvet, M., \& Monnereau, M. (2017). Scattering attenuation profile of the Moon: Implications for shallow moonquakes and the structure of the megaregolith. Physics of the Earth and Planetary Interiors, 262, 28-40.

Gupta, S., Kumar, A., Shukla, A., Suresh, G., \& Baidya, P. (2006). Coda q in the kachchh basin, western india using aftershocks of the bhuj earthquake of january 26 , 2001. pure and applied geophysics, 163(8), 1583-1595.

Gupta, S., Teotia, S., Rai, S., \& Gautam, N. (1998). Coda q estimates in the koyna region, india. $\quad$ In $Q$ of the earth: Global, regional, and laboratory studies (pp. 713731). Springer.

Hoshiba, M. (1993). Separation of scattering attenuation and intrinsic absorption in japan using the multiple lapse time window analysis of full seismogram envelope. Journal of geophysical research, 98(B9), 15809-15824. 
Ibanez, J., Del Pezzo, E., De Miguel, F., Herraiz, M., Alguacil, G., \& Morales, J. (1990). Depth-dependent seismic attenuation in the granada zone (southern spain). Bulletin of the Seismological Society of America, 80(5), 1232-1244.

InSight Mars SEIS Data Service. (2019). SEIS raw data, InSight Mission. IPGP, JPL, CNES, ETHZ, ICL, MPS, ISAE-Supaero, LPG, MFSC. Retrieved from http://dx.doi.org/10.18715/SEIS. INSIGHT .XB_2016 doi: http://dx.doi.org/ 10.18715/SEIS.INSIGHT.XB_2016

Insight Mars SEIS Data Service. (2019a). Insight seis data bundle. PDS Geosciences (GEO) Node. Retrieved from https://pds.jpl.nasa.gov/ds-view/pds/ viewBundle.jsp?identifier=urn:nasa:pds:insight_seis\&version=1.1 doi: 10 $.17189 / 1517570$

InSight Marsquake Service. (2020). Mars Seismic Catalogue, InSight Mission; V1 2/1/2020. ETHZ, IPGP, JPL, ICL, ISAE-Supaero, MPS, Univ Bristol. Dataset. doi: https://doi.org/10.12686/a6

InSight SEIS Science Team. (2019). InSight SEIS Data Bundle. Retrieved from https://pds-geosciences.wustl.edu/missions/insight/seis.htm

Jacobson, R. (1987). An investigation into the fundamental relationships between attenuation, phase dispersion, and frequency using seismic refraction profiles over sedimentary structures. Geophysics, 52(1), 72-87.

KANAO, M., \& ITO, K. (1991). Attenuation of s waves and coda waves in the inner zone of southwestern japan. Bulletin of the Disaster Prevention Research Institute, 41(2), 87-107.

Knapmeyer-Endrun, B., Panning, M. P., Bissig, F., Joshi, R., Khan, A., Kim, D., ... others (2021). Thickness and structure of the martian crust from insight seismic data. Science, 373(6553), 438-443.

Korn, M. (1990). A modified energy flux model for lithospheric scattering of teleseismic body waves. Geophysical Journal International, 102, 165-175. 
Kosuga, M. (1992). Dependence of coda q on frequency and lapse time in the western nagano region, central japan. Journal of Physics of the Earth, 40(2), 421-445.

Kubanza, M., Nishimura, T., \& Sato, H. (2007). Evaluation of strength of heterogeneity in the lithosphere from peak amplitude analyses of teleseismic short-period vector P waves. Geophysical Journal International, 171(1), 390-398.

Kumar, C. P., Sarma, C., Shekar, M., \& Chadha, R. (2007). Attenuation studies based on local earthquake coda waves in the southern indian peninsular shield. Natural Hazards, 40(3), 527-536.

Latham, G., Ewing, M., Dorman, J., Lammlein, D., Press, F., Toksőz, N., ... Nakamura, Y. (1972). Moonquakes and lunar tectonism. The Moon, 4(3-4), 373-382. Retrieved from www.scopus.com (Cited By :25)

Latham, G., Ewing, M., Press, F., Sutton, G., Dorman, J., Nakamura, Y., ... Duennebier, F. (1970). Passive seismic experiment. Science, 167(3918), 455-457.

Lognonné, P., Banerdt, W., Pike, W., Giardini, D., Christensen, U., Garcia, R. F., .. others (2020). Constraints on the shallow elastic and anelastic structure of mars from insight seismic data. Nature Geoscience, 13(3), 213-220.

Lognonné, P., Banerdt, W. B., Giardini, D., Pike, W., Christensen, U., Laudet, P., ... others (2019). Seis: Insight's seismic experiment for internal structure of mars. Space Science Reviews, 215(1).

Lognonné, P., Gagnepain-Beyneix, J., \& Chenet, H. (2003). A new seismic model of the moon: Implications for structure, thermal evolution and formation of the moon. Earth and Planetary Science Letters, 211, 27.

Madariaga, R. (1976). Dynamics of an expanding circular fault. Bulletin of the Seismological Society of America, 66(3), 639-666.

Margerin, L. (2017). Computation of Green's function of 3-D radiative transport equations for non-isotropic scattering of $\mathrm{P}$ and unpolarized $\mathrm{S}$ waves. $\quad$ Pure and $A p$ - 
plied Geophysics, $174(11)$, 4057-4075.

Margerin, L., Campillo, M., \& van Tiggelen, B. A. (2000). Monte Carlo simulation of multiple scaterring of elastic waves. Journal of Geophysical Research, 105, 78737892.

Mayeda, K., Koyanagi, S., Hoshiba, M., Aki, K., \& Zeng, Y. (1992). A comparative study of scattering, intrinsic, and coda $\mathrm{Q}^{-1}$ for Hawaii, Long Valley, and central California between 1.5 and $15.0 \mathrm{~Hz}$. Journal of Geophysical Research: Solid Earth, $97(\mathrm{~B} 5), 6643-6659$.

Mitchell, B. J. (1995). Anelastic structure and evolution of the continental crust and upper mantle from seismic surface wave attenuation. Reviews of Geophysics, 33(4), 441-462. Retrieved from www.scopus.com (Cited By :212)

Mitchell, B. J., Cong, L., \& Ekström, G. (2008). A continent-wide map of 1-hz lg coda q variation across eurasia and its relation to lithospheric evolution. Journal of Geophysical Research: Solid Earth, 113(B4).

Nakamura, Y. (1976). Seismic energy transmission in the lunar surface zone determined from signals generated by movement of lunar rovers. Bulletin of the Seismological Society of America, 66, 593-608.

Nakamura, Y., Latham, G., \& Dorman, J. (1982). Apollo lunar seismic experimentfinal summary. In Proc. lunar planet. sci. conf. 13th.

Przybilla, J., Wegler, U., \& Korn, M. (2009). Estimation of crustal scattering parameters with elastic radiative transfer theory. Geophysical Journal International, $178(2), 1105-1111$.

Rautian, T., \& Khalturin, V. (1978). The use of the coda for determination of the earthquake source spectrum. Bulletin of the Seismological Society of America, 68(4), 923-948.

Romanowicz, B., \& Michell, B. (2015). Deep earth structure: Q of the earth from 
crust to core. In G. Schubert (Ed.), Treatise on geophysics (pp. 789-827). Elsevier.

Ryzhik, L., Papanicolaou, G., \& Keller, J. B. (1996). Transport equation for elastic and other waves in random media. Wave Motion, 24, 327-370.

Saito, T., Sato, H., \& Ohtake, M. (2002). Envelope broadening of spherically outgoing waves in three-dimensional random media having power law spectra. Journal of Geophysical Research, 107, 2089.

Sanborn, C. J., Cormier, V. F., \& Fitzpatrick, M. (2017). Combined effects of deterministic and statistical structure on high-frequency regional seismograms. Geophysical Journal International, 210(2), 1143-1159.

Sato, H. (1982). Amplitude attenuation of impulsive waves in random media based on travel time corrected mean wave formalism. The Journal of the Acoustical Society of America, 71, 559 .

Sato, H. (1984). Attenuation and envelope formation of three-component seismograms of small local earthquakes in randomly inhomogeneous lithosphere. Journal of Geophysical Research, 89(B2), 1221-1241.

Sato, H. (1989). Broadening of Seismogram Envelopes in the Randomly Inhomogeneous lithosphere Based on the Parabolic Approximation: Southeastern Honshu, Japan. J. Geophys. Res, 94, 17735-17747.

Sato, H. (2019). Power spectra of random heterogeneities in the solid earth. Solid Earth, 10(1), 275-292.

Sato, H., Fehler, M. C., \& Maeda, T. (2012). Seismic wave propagation and scattering in the heterogeneous earth (Vol. 496). Springer.

Scheller, E. L., Ehlmann, B. L., Hu, R., Adams, D. J., \& Yung, Y. L. (2021). Longterm drying of mars by sequestration of ocean-scale volumes of water in the crust. Science. Retrieved from https://science.sciencemag.org/content/early/2021/ 03/15/science.abc7717 doi: 10.1126/science.abc7717 
Scholz, J.-R., Widmer-Schnidrig, R., Davis, P., Lognonné, P., Pinot, B., Garcia, R. F., .. others (2020). Detection, analysis, and removal of glitches from insight's seismic data from mars. Earth and Space Science, 7(11), e2020EA001317.

Shearer, P., \& Earle, P. (2004). The global short-period wavefield modelled with a Monte Carlo seismic phonon method. Geophysical Journal International, 158(3), 1103-1117.

Singh, S., Garcia, D., Pacheco, J., Valenzuela, R., Bansal, B., \& Dattatrayam, R. (2004). Q of the indian shield. Bulletin of the Seismological Society of America, $94(4), 1564-1570$.

Singh, S., \& Herrmann, R. B. (1983). Regionalization of crustal coda q in the continental united states. Journal of Geophysical Research: Solid Earth, 88(B1), 527538.

Takahashi, T., Sato, H., Nishimura, T., \& Obara, K. (2007). Strong inhomogeneity beneath Quaternary volcanoes revealed from the peak delay analysis of S-wave seismograms of microearthquakes in northeastern Japan. Geophysical Journal International, 168(1), 90-99.

Takahashi, T., Sato, H., Nishimura, T., \& Obara, K. (2009). Tomographic inversion of the peak delay times to reveal random velocity fluctuations in the lithosphere: method and application to northeastern Japan. Geophysical Journal International, 178, 1437-1455.

Takeuchi, N. (2016). Differential monte carlo method for computing seismogram envelopes and their partial derivatives. Journal of Geophysical Research: Solid Earth, $121(5), 3428-3444$.

Tittman, B. (1977). Lunar rock q in 3000-5000 range achieved in laboratory. Philosophical Transactions of the Royal Society of London. Series A, Mathematical and Physical Sciences, 285(1327), 475-479.

Tittmann, B., Clark, V., Richardson, J., \& Spencer, T. (1980). Possible mecha- 
nism for seismic attenuation in rocks containing small amounts of volatiles. Journal of Geophysical Research: Solid Earth, 85(B10), 5199-5208.

Turner, J. (1998). Scattering and diffusion of seismic waves. Bulletin of the Seismological Society of America, 88, 276-283.

Turner, J., \& Weaver, R. L. (1994). Radiative transfer of ultrasound in a polycrystal. Journal of the Acoustical Society of America, 96, 3675-3683.

Tuvè, T., Bianco, F., Ibáñez, J., Patanè, D., Del Pezzo, E., \& Bottari, A. (2006). Attenuation study in the straits of messina area (southern italy). Tectonophysics, 421(3-4), 173-185.

van Driel, M., Ceylan, S., Clinton, J. F., Giardini, D., Horleston, A., Margerin, L., ... Banerdt, W. B. (2021). High-frequency seismic events on mars observed by insight. Journal of Geophysical Research: Planets, 126(2), e2020JE006670. Retrieved from https://agupubs.onlinelibrary.wiley.com/doi/abs/10.1029/ 2020JE006670 (e2020JE006670 2020JE006670) doi: https://doi.org/10.1029/ 2020JE006670

Weaver, R. L. (1990). Diffusivity of ultrasound in polycrystals. J. Mech. Phys. Solids, $38,55-86$.

Woodgold, C. (1994). Coda q in the charlevoix, quebec, region: lapse-time dependence and spatial and temporal comparisons. Bulletin of the Seismological Society of America, 84(4), 1123-1131.

Woodgold, C. R. (1990). Estimation of q in eastern canada using coda waves. Bulletin of the Seismological Society of America, 80(2), 411-429.

Xie, J., \& Mitchell, B. (1990). A back-projection method for imaging large-scale lateral variations of $\lg$ coda q with application to continental africa. Geophysical Journal International, 100(2), 161-181.

Zuber, M. T., Smith, D. E., Watkins, M. M., Asmar, S. W., Konopliv, A. S., 
936
Lemoine, F. G., ... others

(2013).

Gravity field of the moon from the gravity recovery and interior laboratory (grail) mission. Science, 339(6120), 668-671.

Zweifel, P., Mance, D., ten Pierick, J., Giardini, D., Schmelzbach, C., Haag, T., ...

Banerdt, W. B. (submitted). The noise model of the seis seismometer of the insight mission to mars. submitted to BSSA. 


\section{Authors mailing list}

- Sabrina Menina: menina@ipgp.fr, Institut de Physique du Globe de Paris - 1 rue Jussieu - 75238 Paris cedex 05, France

- Ludovic Margerin: Ludovic.Margerin@irap.omp.eu, Institut de Recherches en Astrophysique et Planétologie - 14, Avenue Edouard Belin, 31400 Toulouse, France

- Taïchi Kawamura: kawamura@ipgp.fr, Institut de Physique du Globe de Paris - 35 rue Hélène Brion 75205 PARIS CEDEX 13, France

- Philippe Lognonné: lognonne@ipgp.fr, Institut de Physique du Globe de Paris - 35 rue Hélène Brion - Case 7071, Lamarck A - 75205 Paris Cedex 13, France

- Jules Marti: Jules.MARTI@student.isae-supaero.fr, Institut de Recherches en Astrophysique et Planétologie - 14, Avenue Edouard Belin, 31400 Toulouse, France

- Mélanie Drilleau: Melanie.DRILLEAU@isae-supaero.fr, Institut Supérieur de l'Aéronautique et de l'Espace, 10, avenue Édouard-Belin BP 54032 - 31055 Toulouse CEDEX 4, France

- Marie Calvet: Marie.Calvet@irap.omp.eu, Institut de Recherches en Astrophysique et Planétologie, Observatoire MidiPyrénées, 11 avenue Edouard Belin 31400 Toulouse, France

- Nicolas Compaire: Nicolas.COMPAIRE@isae-supaero.fr, Institut Supérieur de l'Aéronautique et de l'Espace, 10, avenue Édouard-Belin BP 54032 - 31055 Toulouse CEDEX 4, France 
- Raphaël Garcia: Raphael.GARCIA@isae-supaero.fr, Institut Supérieur de l'Aéronautique et de l'Espace, 10, avenue Édouard-Belin BP 54032 - 31055 Toulouse CEDEX 4, France

- Foivos Karakostas: foivos.karakostas@ingv.it, Istituto Nazionale di Geofisica e Vulcanologia, Sezione di Bologna, Via Donato Creti, 12, Bologna, 40128, ITALY

- Nicholas Schmerr: nschmerr@umd.edu University of Maryland, Department of Geology, 8000 Regents Dr,College Park, MD, 20742, USA

- Martin van Driel: vandriel@erdw.ethz.ch ETH Zürich, Department of Earth Sciences, Sonneggstrasse 5, 8092 Zürich, Switzerland

- Simon C. Stähler: simon.staehler@erdw.ethz.ch ETH Zürich, Department of Earth Sciences, Sonneggstrasse 5, 8092 Zürich, Switzerland

- Matthieu Plasman: plasman@ipgp.fr, Institut de Physique du Globe de Paris - 1 rue Jussieu - 75238 Paris cedex 05

• Domenico Giardini: domenico.giardini@erdw.ethz.ch, ETH Zürich, Department of Earth Sciences, Sonneggstrasse 5, 8092 Zürich, Switzerland

- Sebastian Carrasco: acarrasc@uni-koeln.de, Université de Cologne, Albertus-Magnus-Platz, 50923 Köln, Allemagne

- Brigitte Knapmeyer-Endrun: brigitte.knapmeyer-endrun@uni-koeln.de, Université de Cologne, Albertus-Magnus-Platz, 50923 Köln, Allemagne 
990

996

- Grégory Sainton: sainton@ipgp.fr, Institut de Physique du Globe de Paris - 1 rue Jussieu - 75238 Paris cedex 05

- William T. Pike: w.t.pike@imperial.ac.uk, Imperial College London, Exhibition Road, London SW7 2AZ, United Kingdom

-William B. Banerdt: william.b.banerdt@jpl.nasa.gov, Jet Propulsion Laboratory, 4800 Oak Grove Drive, M/S 321-B60, Pasadena, CA 91109, USA 


\section{Tables}

Table 1: List of events analyzed in this study. $t_{p, s} \quad$ : arrival time of primary, secondary arrival (expressed in Universal Time Coordinated). The coda start time is determined empirically and corresponds to the observation of steady decay long after the passage of the secondary wavetrain. The coda end time is determined by the $\mathrm{S} / \mathrm{N}$ ratio (always greater than 2). The distance range corresponds to the assumptions $c_{s}=3.0 \mathrm{~km} / \mathrm{s}, \mathrm{cp} / \mathrm{cs}=1.85$ and $c_{s}=1.7 \mathrm{~km} / \mathrm{s}, c_{p} / c_{s}=1.65$.

\begin{tabular}{llllllll}
\hline Event & Type & Date & $t_{p}$ & $t_{s}$ & $\begin{array}{l}\text { Coda } \\
\text { start time }\end{array}$ & $\begin{array}{l}\text { Coda } \\
\text { end time }\end{array}$ & $\begin{array}{l}\text { Distance range } \\
(\mathrm{km})\end{array}$ \\
& & & & & & & \\
\hline S0239a & HF & $2019 / 07 / 30$ & $14: 16: 59$ & $14: 21: 46$ & $14: 23: 06$ & $14: 25: 36$ & {$[1243,1881]$} \\
S0260a & HF & $2019 / 08 / 21$ & $05: 11: 21$ & $05: 15: 53$ & $05: 18: 43$ & $05: 22: 33$ & {$[1180,1785]$} \\
S0291c & HF & $2019 / 09 / 22$ & $03: 46: 14$ & $03: 49: 58$ & $03: 52: 38$ & $03: 54: 18$ & {$[971,1469]$} \\
S0304b & HF & $2019 / 10 / 05$ & $12: 47: 55$ & $12: 51: 54$ & $12: 54: 24$ & $12: 56: 54$ & {$[1027,1554]$} \\
S0308a & HF & $2019 / 10 / 09$ & $15: 45: 53$ & $15: 50: 28$ & $15: 53: 08$ & $15: 55: 48$ & {$[1187,1796]$} \\
S0314b & HF & $2019 / 10 / 15$ & $15: 41: 40$ & $15: 48: 58$ & $15: 51: 38$ & $15: 54: 38$ & {$[1053,1593]$} \\
S0319b & HF & $2019 / 10 / 20$ & $17: 44: 55$ & $17: 48: 30$ & $17: 49: 50$ & $17: 52: 50$ & {$[1230,1861]$} \\
S0327c & HF & $2019 / 10 / 29$ & $04: 03: 21$ & $04: 06: 40$ & $04: 08: 40$ & $04: 11: 20$ & {$[859,1300]$} \\
S0331a & HF & $2019 / 11 / 01$ & $08: 40: 32$ & $08: 44: 15$ & $08: 46: 55$ & $08: 49: 55$ & {$[1054,1594]$} \\
S0343b & HF & $2019 / 11 / 14$ & $11: 59: 43$ & $12: 04: 49$ & $12: 06: 49$ & $12: 09: 09$ & {$[1321,1998]$} \\
S0352a & HF & $2019 / 11 / 23$ & $18: 18: 46$ & $18: 22: 56$ & $18: 24: 36$ & $18: 27: 16$ & {$[1208,1828]$} \\
S0384b & HF & $2019 / 12 / 26$ & $13: 10: 25$ & $13: 13: 22$ & $13: 16: 02$ & $13: 18: 42$ & {$[807,1221]$} \\
S0392a & HF & $2020 / 01 / 03$ & $17: 25: 23$ & $17: 29: 30$ & $17: 32: 10$ & $17: 34: 40$ & {$[1213,1835]$} \\
S0128a & VF & $2019 / 04 / 07$ & $09: 33: 26$ & $09: 34: 50$ & $09: 36: 50$ & $09: 41: 50$ & {$[363,550]$} \\
S0263a & VF & $2019 / 08 / 23$ & $11: 09: 30$ & $11: 10: 26$ & $11: 11: 46$ & $11: 14: 06$ & {$[240,364]$} \\
S0264e & VF & $2019 / 08 / 25$ & $10: 31: 20$ & $10: 37: 24$ & $10: 39: 24$ & $10: 43: 34$ & {$[1571,2377]$} \\
S0334a & VF & $2019 / 11 / 05$ & $04: 55: 03$ & $04: 58: 23$ & $05: 00: 03$ & $05: 04: 13$ & {$[863,1308]$} \\
S0421a & VF & $2020 / 02 / 02$ & $17: 38: 08$ & $17: 44: 56$ & $17: 47: 16$ & $17: 50: 16$ & {$[1762,2667]$} \\
S0424c & VF & $2020 / 02 / 05$ & $17: 29: 56$ & $17: 35: 32$ & $17: 37: 32$ & $17: 41: 22$ & {$[1450,2194]$} \\
S0734a & VF & $2020 / 12 / 19$ & $10: 14: 00$ & $10: 15: 21$ & $10: 17: 01$ & $10: 19: 31$ & {$[348,527]$} \\
S0756a & VF & $2021 / 01 / 11$ & $01: 12: 07$ & $01: 15: 07$ & $01: 17: 27$ & $01: 21: 37$ & {$[777,1176]$} \\
& & & & & & &
\end{tabular}


Table 2: Geographical location and reference associated to the $Q_{c}$ measurements shown in Figure 9.

\begin{tabular}{|c|c|c|}
\hline Label & Location & Articles \\
\hline 1 & Wakayama Shallow, Japan (40 - 45 s) & (KANAO \& ITO, 1991) \\
\hline 2 & Nagoya Shallow, Japan (50 - $60 \mathrm{~s})$ & (KANAO \& ITO, 1991) \\
\hline 3 & Western Nagano, Japan & (Kosuga, 1992) \\
\hline 4 & Kanto-Tokai, Japan (V. compo. average) & (Fehler et al., 1992) \\
\hline 5 & Granada zone, southern Spain (90 s) & (Ibanez et al., 1990) \\
\hline 6 & Southern Sicily, Italy (50 s) & (Giampiccolo et al., 2004) \\
\hline 7 & Southern Apennines zone, Italy & (Bianco et al., 2002) \\
\hline 8 & Konya region, India (40 s) & (Gupta et al., 1998) \\
\hline 9 & Kachchh Basin, western India (50 - 80 s) & (Gupta et al., 2006) \\
\hline 10 & Long Valley, California & (Mayeda et al., 1992) \\
\hline 11 & Central California & (Mayeda et al., 1992), \\
\hline 12 & Hawaii & (Mayeda et al., 1992) \\
\hline 13 & Straits of Messina area, southern Italy (60 s) & (Tuvè et al., 2006) \\
\hline 14 & Kothagudem, southern India & (Kumar et al., 2007) \\
\hline 15 & Cuddapah, southern India & (Kumar et al., 2007) \\
\hline 16 & Dharwar, southern India & (Kumar et al., 2007) \\
\hline 17 & Singhbhum Odisha craton, eastern India & (Biswas et al., 2019) \\
\hline 18 & Chotanagpur granitic gneissic terrain, eastern India & (Biswas et al., 2019) \\
\hline 19 & Eastern Ghat mobile belt, eastern India & (Biswas et al., 2019) \\
\hline 20 & Continental United States & (Singh \& Herrmann, 1983) \\
\hline 21 & New Brunswick, eastern Canada & (C. R. Woodgold, 1990) \\
\hline 22 & Near Ottawa, eastern Canada & (C. R. Woodgold, 1990) \\
\hline 23 & Adirondack Mountains, eastern Canada & (C. R. Woodgold, 1990) \\
\hline 24 & near Val-d'Or, Quebec, eastern Canada & (C. R. Woodgold, 1990) \\
\hline 25 & St. Lawrence River, eastern Canada & (C. R. Woodgold, 1990) \\
\hline 26 & Indian shield & (Singh et al., 2004) \\
\hline 27 & East European shield & (Mitchell et al., 2008) \\
\hline 28 & Siberian shield & (Mitchell et al., 2008) \\
\hline 29 & Arabian shield & (Mitchell et al., 2008) \\
\hline 30 & Northwest of Africa & (Xie \& Mitchell, 1990) \\
\hline 31 & Northeast of Africa (Sahara) & (Xie \& Mitchell, 1990) \\
\hline 32 & Kalahari Craton & (Xie \& Mitchell, 1990) \\
\hline 33 & Brazilian shield & (De Souza \& Mitchell, 1998) \\
\hline 34 & InSight & Event S0734a $(\alpha=0)$ \\
\hline 35 & InSight & Event S0734a $(\alpha=1)$ \\
\hline 36 & InSight & VF events $(\alpha=0)$ \\
\hline 37 & InSight & VF events $(\alpha=1)$ \\
\hline 38 & Apollo mission (The Moon) & (Gillet et al., 2017) \\
\hline
\end{tabular}




\section{List of Figure Captions}

- Figure 1 (a) Waveform filtered at $3.5 \mathrm{~Hz}$ (top) and spectrogram (bottom) of the vertical component of the S0334 VF event recorded by the VBB sensor at 20 samples per seconds. The arrival times of the first and second arrivals are denoted by $t_{p}$ and $t_{s}$ respectively and are indicated with red dashed lines. Time $t=0$ coincides with the onset of the first arrival. (b) Same as (a) for the HF event S0314b.

- Figure 2 Spectral amplitude (m/Hz) as a function of frequency: (a) total spectral amplitude, (b) vertical component spectral amplitude, (c) horizontal components spectral amplitude for HF events (blue) and VF events (red). At each frequency, the mean energy is measured on the vertical and horizontal components in a time window of $200 \mathrm{~s}$ duration starting after the second arrival. The RMS amplitude has been normalized by the bandwidth.

- Figure 3 Envelope characteristics of the VF event S0734a. The data were collected on the VBB sensors with sampling rates of 20 sps. (a): Base 10 logarithm of energy envelopes normalized by the noise level as a function of time and fit of the coda decay (black dashed lines). The gray dashed line indicates $S / N=2$. The different colors correspond to different frequency bands indicated in inset. The time $\mathrm{t}=0 \mathrm{~s}$ correspond to the first arrival. $t_{p}$ and $t_{s}$ refer to the arrival times of the first and second wave packets (red dashed lines). The black dots show the arrival times of the energy maximum $\left(t_{\max }\right)$. The magenta and purple dashed lines indicate the mean and standard deviation of $t_{\max }$ in the $2.5-7.5 \mathrm{~Hz}$ band. (b) Frequency dependence of $Q_{c}$ in the 2.5 $7.5 \mathrm{~Hz}$ frequency band. The color bar indicates the $S / N$ ratio measured on the horizontal components. The dashed line shows the power-law fit $Q_{c}=440 f^{1.08}$ ( $f$ is the frequency). (c) Delay time $\left(t_{d}=t_{\max }-t_{s}\right.$ ) as a function of frequency. 
The magenta and purple dashed lines show the mean and standard deviation of $t_{d}$ in the $2.5-7.5 \mathrm{~Hz}$ band.

- Figure 4 Envelope characteristics of a regional event $\left(m_{b}=4.0\right)$ recorded by a VBB seismometer (Streckheisen STS-1) on the 2007-07-16 at station FRB (Iqaluit, Canada). The quake location is $65.11^{\circ} \mathrm{N}, 86.81^{\circ} \mathrm{W}$, corresponding to an epicentral distance of approximately 900km (information provided by IRISDMC). (a)-(c): same as Figure 3 except for the power law fit $Q_{c}=740 f^{0.49}$.

- Figure 5 Horizontal envelope characteristics of 13 HF events (blue) and 8 VF events (green). Left: delay-time measurements $\left(t_{d}\right)$ as a function of traveltime difference between second and first arrivals $\left(t_{s}-t_{p}\right)$. Right: $Q_{c}$ measurements as a function of $t_{s}-t_{p}$ at $3.5 \mathrm{~Hz}$. Dark and light colours correspond to the choice $\alpha=0$ and $\alpha=1$ in Eq. (1), respectively.

- Figure 6 Coda quality factor $Q_{c}$ as a function of the mean signal-to-noise ratio $(S / N)$ in the $2.5-7.5 \mathrm{~Hz}$ frequency band for $13 \mathrm{HF}$ events (blue) and $8 \mathrm{VF}$ events (green). The measurements were performed on the horizontal components. Dark and light colours correspond to the choice $\alpha=0$ and $\alpha=1$ in Eq. (1), respectively. The mean $S / N$ ratio is calculated in a window of $200 \mathrm{~s}$ duration after the direct S-wave onset. The black dashed line refer to $S / N=2$.

- Figure 7 Noise sensitivity analysis applied to the VF event S0334a. Top: Coda quality factor as a function of the signal-to-noise ratio $(S / N)$ at $2.5 \mathrm{~Hz}$ (left) and $7.5 \mathrm{~Hz}$ (right). $S / N$ measured are calculated in a window of $200 \mathrm{~s}$ duration starting at the second arrival onset. Noise data recorded at the same LMST (Local Mean Solar Time) on Sol 333 has been added to the data. In inset, we indicate the amplitude factor $k$ applied to the noise prior to addition to the signal $(k \in\{0,1,2,3,4,5\})$. 
- Figure $8 Q_{0}$ and $n$ computed as a function of $t_{s}-t_{p}$ for the $8 \mathrm{VF}$ events selected. Dark and light colours correspond to the choice $\alpha=0$ and $\alpha=1$ in Eq. (1), respectively. The dashed lines refers to the mean values for $\alpha=0\left(Q_{0}\right.$ $=644$ and $n=1.04)$ and $\alpha=1\left(Q_{0}=701\right.$ and $\left.n=1.038\right)$.

- Figure 9 Coda attenuation $Q_{c}^{-1}$ as a function of the frequency for different regions on Earth (blue for tectonically active, green-yellow for cratons), the Moon (grey) and Mars (with $\alpha=0$ in red and $\alpha=1$ in orange). The numbers in inset refer to Table 2 where more details can be found on the geographical locations for Earth data, as well as the references of the original publications.

- Figure 10 Radiative transfer modeling of the energy envelope of three VF events: (a) S0734a, (b) S0756a and (c) S0421a VF. The dashed black lines correspond to the energy envelope of the horizontal components filtered around $6 \mathrm{~Hz}$. The green and blue lines show the best-fitting radiative transfer models with $\kappa=0.5$ and $\kappa=2$ respectively. In these examples the $c^{p} / c^{s}$ ratio is set to 1.85 and $c^{s}=1.7 \mathrm{~km} / \mathrm{s}$.

- Figure 11 Radiative transfer modeling of the energy envelope of three VF events: (a) S0734a, (b) S0756a and (c) S0421a VF. The dashed black lines correspond to the energy envelope of the horizontal components filtered around $6 \mathrm{~Hz}$. The red and blue lines show the best-fitting radiative transfer models with $c^{p} / c^{s}=1.65$ and $c^{p} / c^{s}=1.85$, respectively. In these examples $\kappa$ is set to 2 and $c^{s}=1.7 \mathrm{~km} / \mathrm{s}$.

- Figure 12 Inversion results for seven VF events. (a) Diffusivity $\left(\mathrm{km}^{2} / \mathrm{s}\right)$, (b) S-wave scattering quality factor and (c) S-wave absorption quality factor as a function of $\left(t_{s}-t_{p}\right)$ for selected VF events. The dashed line shows the best fit to the data assuming a law of the form $y=y_{0} \exp \left(t_{s}-t_{p}\right) / \xi$. 

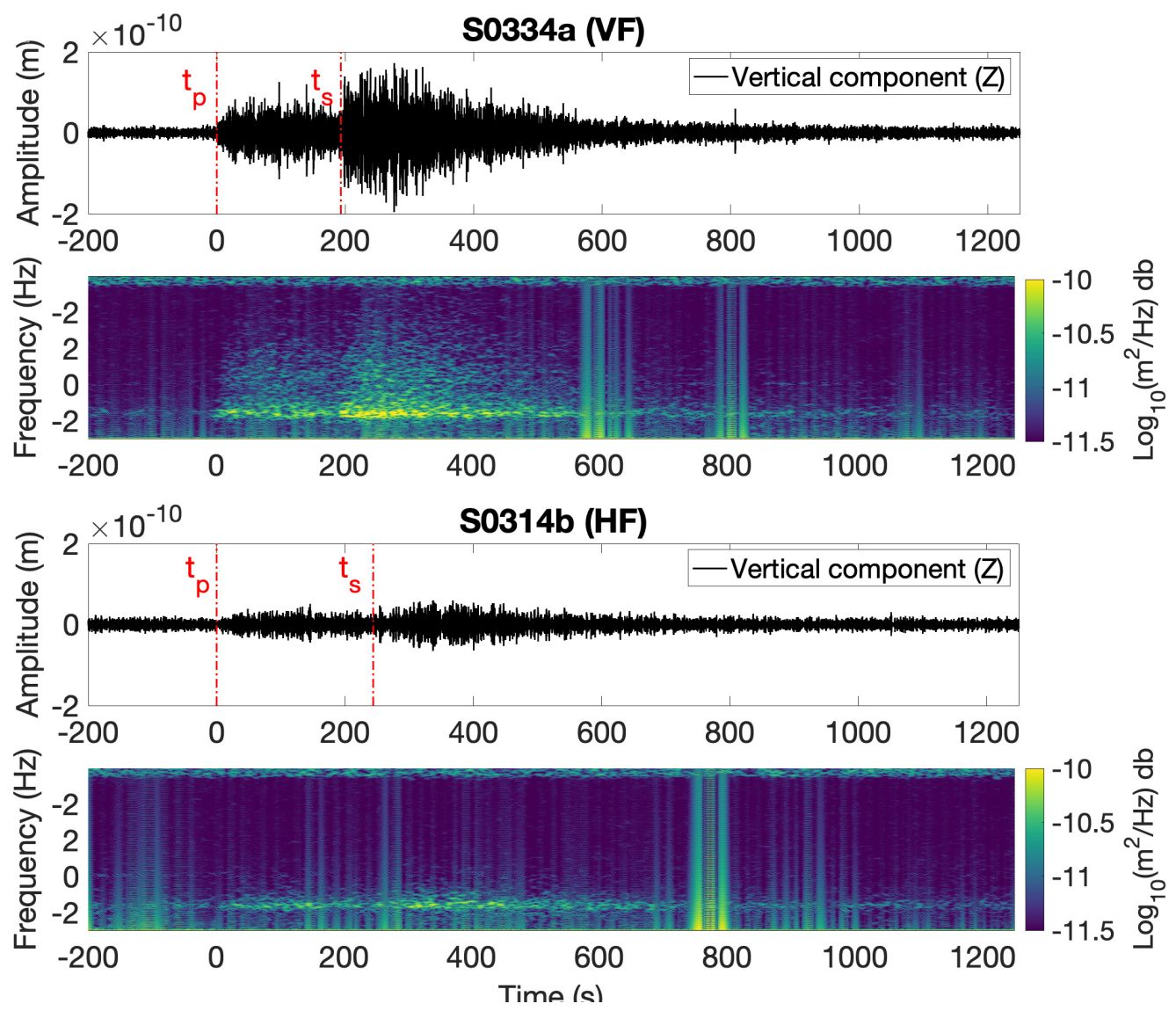

Figure 1: (a) Waveform filtered at $3.5 \mathrm{~Hz}$ (top) and spectrogram (bottom) of the vertical component of the S0334 VF event recorded by the VBB sensor at 20 samples per seconds. The arrival times of the first and second arrivals are denoted by $t_{p}$ and $t_{s}$ respectively and are indicated with red dashed lines. Time $t=0$ coincides with the onset of the first arrival. (b) Same as (a) for the HF event S0314b. 

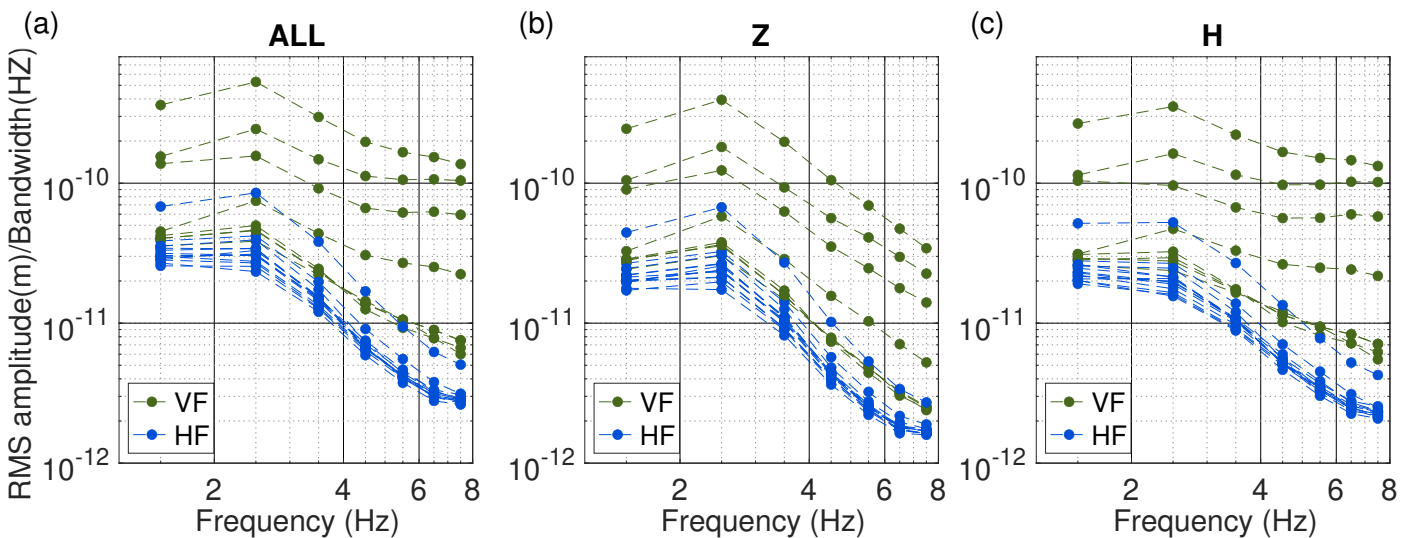

Figure 2: Spectral amplitude $(\mathrm{m} / \mathrm{Hz})$ as a function of frequency: (a) total spectral amplitude, (b) vertical component spectral amplitude, (c) horizontal components spectral amplitude for HF events (blue) and VF events (red). At each frequency, the mean energy is measured on the vertical and horizontal components in a time window of $200 \mathrm{~s}$ duration starting after the second arrival. The RMS amplitude has been normalized by the bandwidth. 


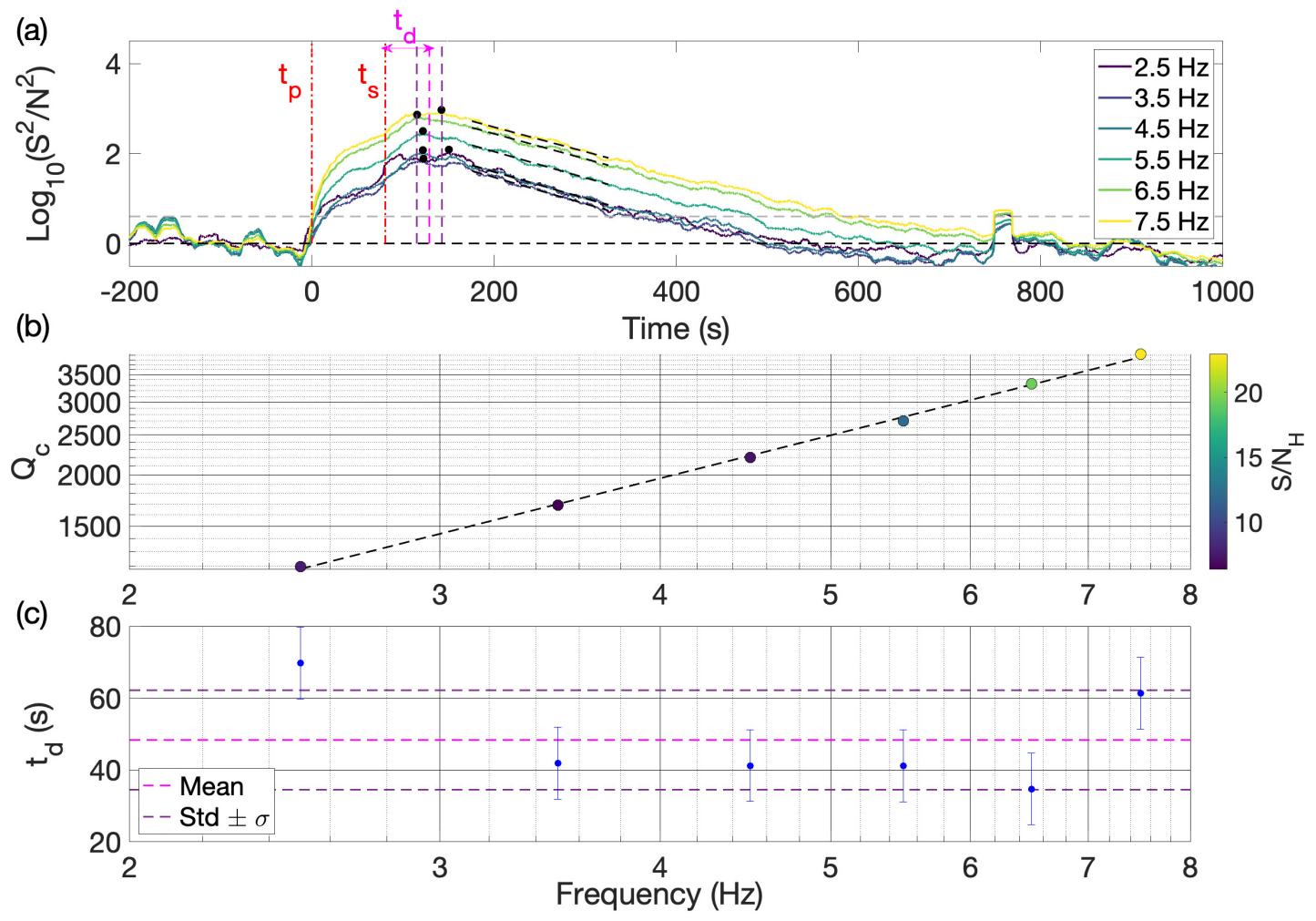

Figure 3: Envelope characteristics of the VF event S0734a. The data were collected on the VBB sensors with sampling rates of 20 sps. (a): Base 10 logarithm of energy envelopes normalized by the noise level as a function of time and fit of the coda decay (black dashed lines). The gray dashed line indicates $S / N=2$. The different colors correspond to different frequency bands indicated in inset. The time $\mathrm{t}=0 \mathrm{~s}$ correspond to the first arrival. $t_{p}$ and $t_{s}$ refer to the arrival times of the first and second wave packets (red dashed lines). The black dots show the arrival times of the energy maximum $\left(t_{\max }\right)$. The magenta and purple dashed lines indicate the mean and standard deviation of $t_{\max }$ in the $2.5-7.5 \mathrm{~Hz}$ band. (b) Frequency dependence of $Q_{c}$ in the $2.5-7.5 \mathrm{~Hz}$ frequency band. The color bar indicates the $S / N$ ratio measured on the horizontal components. The dashed line shows the power-law fit $Q_{c}=440 f^{1.08}$ ( $f$ is the frequency). (c) Delay time $\left(t_{d}=t_{\max }-t_{s}\right)$ as a function of frequency. The magenta and purple dashed lines show the mean and standard deviation of $t_{d}$ in the $2.5-7.5 \mathrm{~Hz}$ band. 


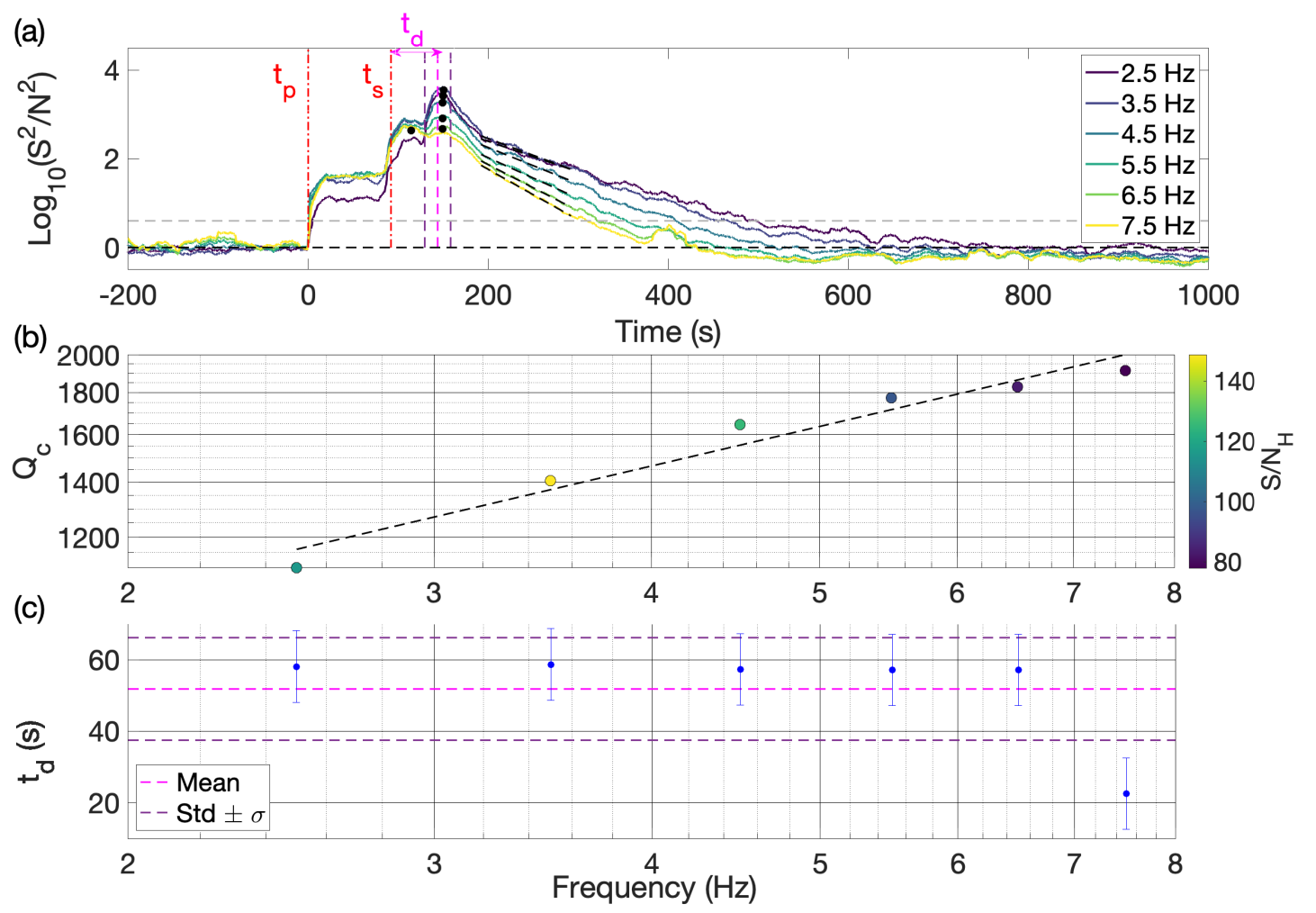

Figure 4: Envelope characteristics of a regional event $\left(m_{b}=4.0\right)$ recorded by a VBB seismometer (Streckheisen STS-1) on the 2007-07-16 at station FRB (Iqaluit, Canada). The quake location is $65.11^{\circ} \mathrm{N}, 86.81^{\circ} \mathrm{W}$, corresponding to an epicentral distance of approximately $900 \mathrm{~km}$ (information provided by IRIS-DMC). (a)-(c): same as Figure 3 except for the power law fit $Q_{c}=740 f^{0.49}$. 

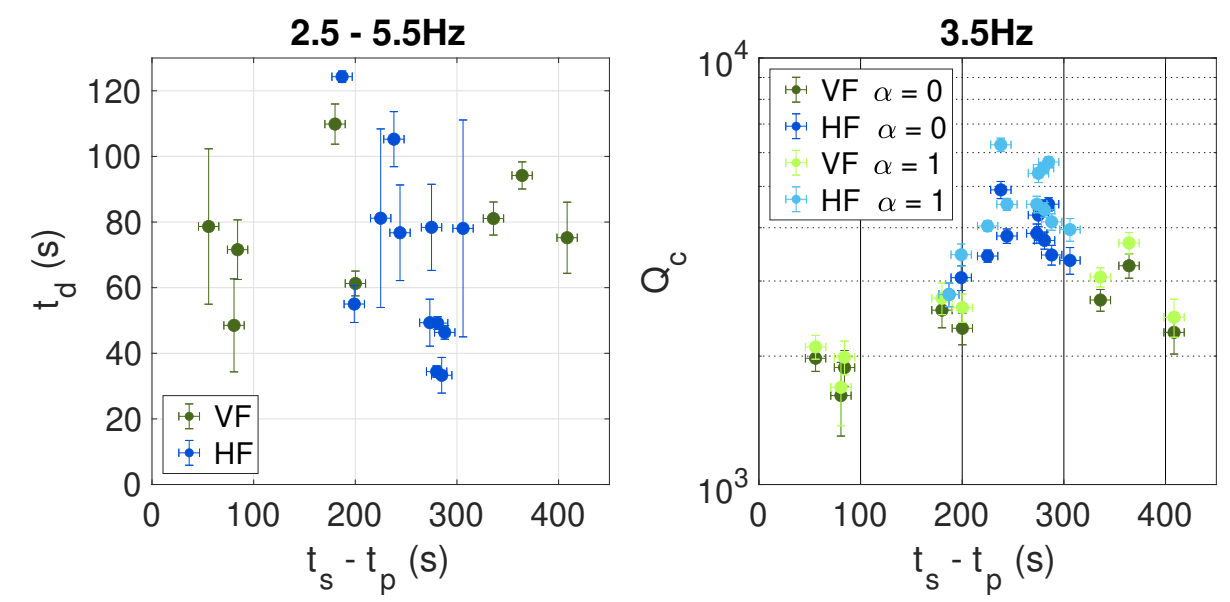

Figure 5: Horizontal envelope characteristics of $13 \mathrm{HF}$ events (blue) and $8 \mathrm{VF}$ events (green). Left: delay-time measurements $\left(t_{d}\right)$ as a function of traveltime difference between second and first arrivals $\left(t_{s}-t_{p}\right)$. Right: $Q_{c}$ measurements as a function of $t_{s}-t_{p}$ at $3.5 \mathrm{~Hz}$. Dark and light colours correspond to the choice $\alpha=0$ and $\alpha=1$ in Eq. (1), respectively. 

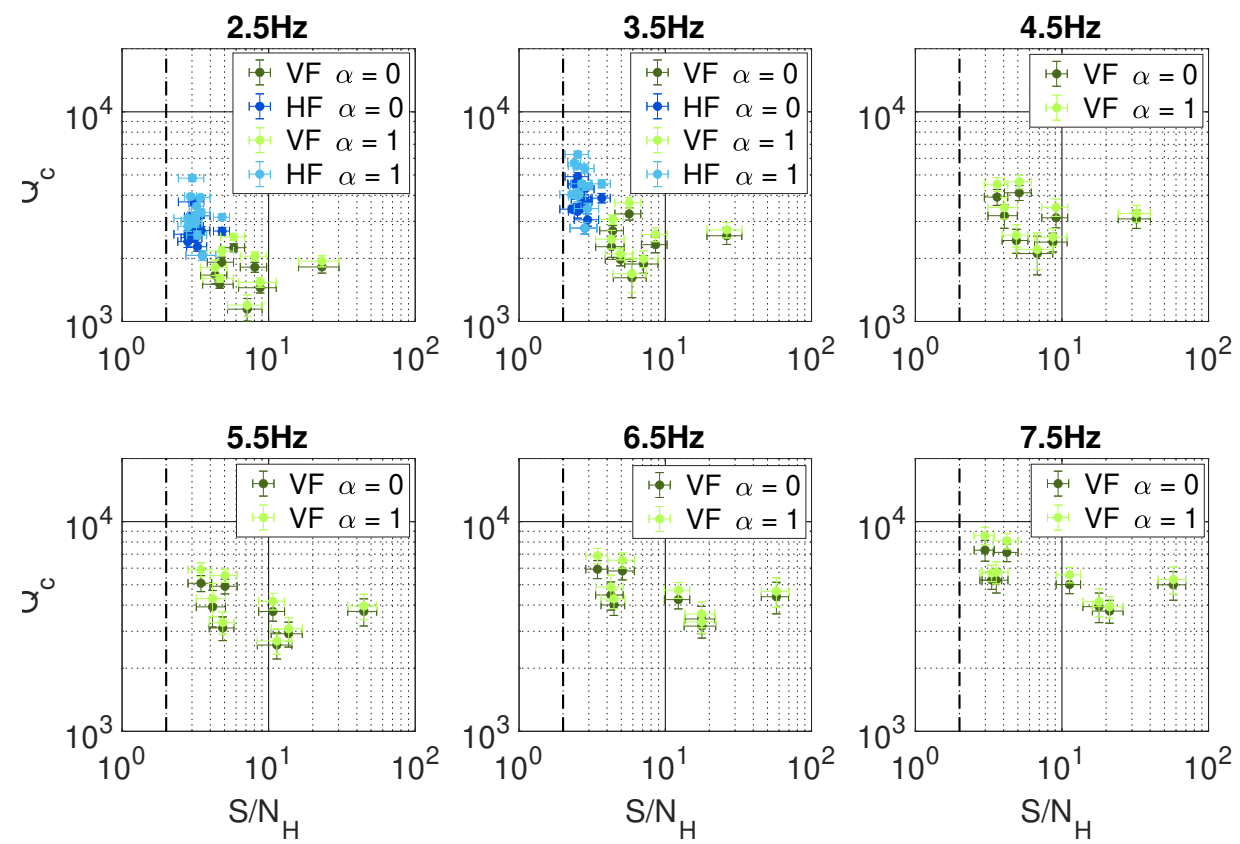

Figure 6: Coda quality factor $Q_{c}$ as a function of the mean signal-to-noise ratio $(S / N)$ in the $2.5-7.5 \mathrm{~Hz}$ frequency band for $13 \mathrm{HF}$ events (blue) and $8 \mathrm{VF}$ events (green). The measurements were performed on the horizontal components. Dark and light colours correspond to the choice $\alpha=0$ and $\alpha=1$ in Eq. (1), respectively. The mean $S / N$ ratio is calculated in a window of $200 \mathrm{~s}$ duration after the direct S-wave onset. The black dashed line refer to $S / N=2$. 

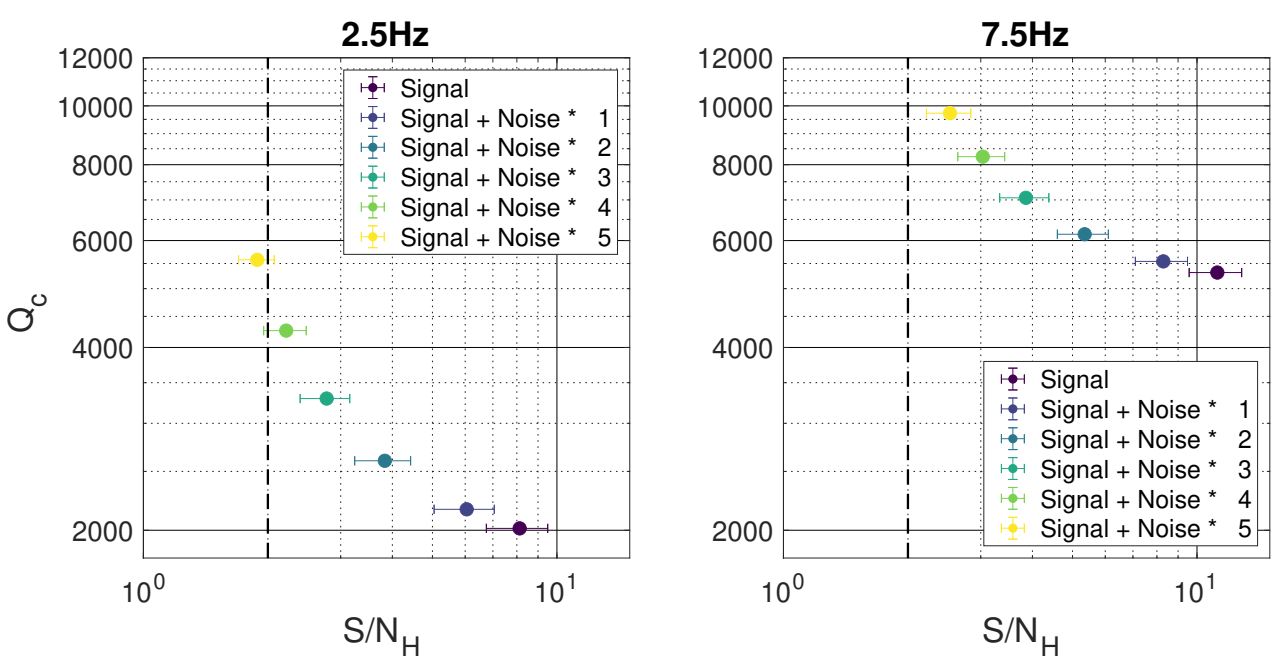

Figure 7: Noise sensitivity analysis applied to the VF event S0334a. Top: Coda quality factor as a function of the signal-to-noise ratio $(S / N)$ at $2.5 \mathrm{~Hz}$ (left) and $7.5 \mathrm{~Hz}$ (right). $S / N$ measured are calculated in a window of $200 \mathrm{~s}$ duration starting at the second arrival onset. Noise data recorded at the same LMST (Local Mean Solar Time) on Sol 333 has been added to the data. In inset, we indicate the amplitude factor $k$ applied to the noise prior to addition to the signal $(k \in\{0,1,2,3,4,5\})$.
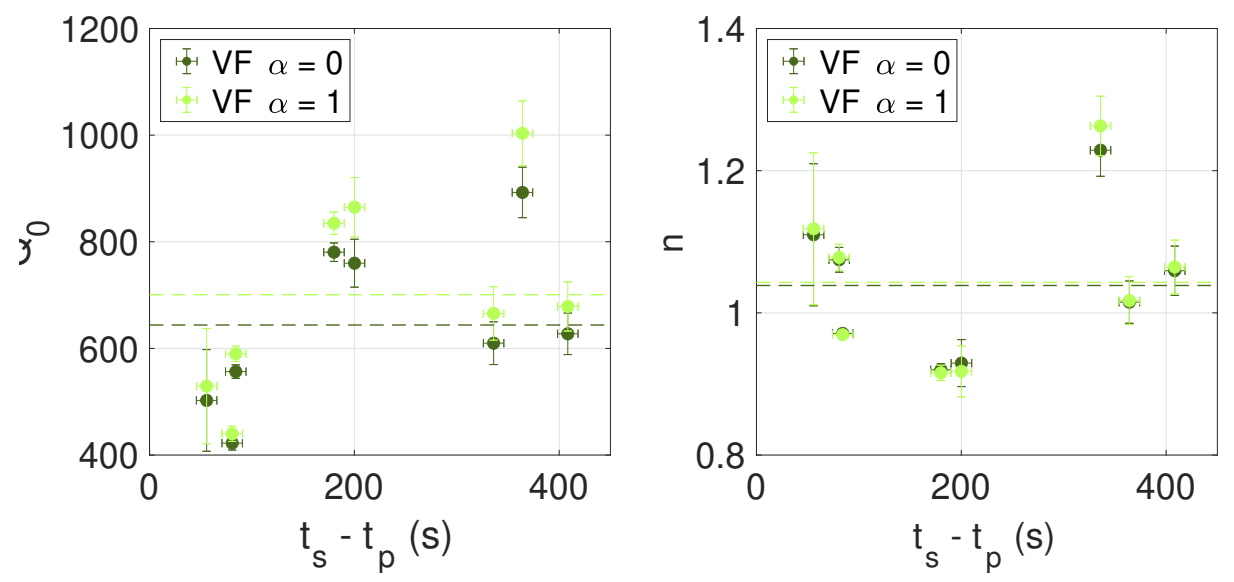

Figure 8: $Q_{0}$ and $n$ computed as a function of $t_{s}-t_{p}$ for the $8 \mathrm{VF}$ events selected. Dark and light colours correspond to the choice $\alpha=0$ and $\alpha=1$ in Eq. (1), respectively. The dashed lines refers to the mean values for $\alpha=0\left(Q_{0}=644\right.$ and $\left.n=1.04\right)$ and $\alpha=1\left(Q_{0}\right.$ $=701$ and $n=1.038$. 


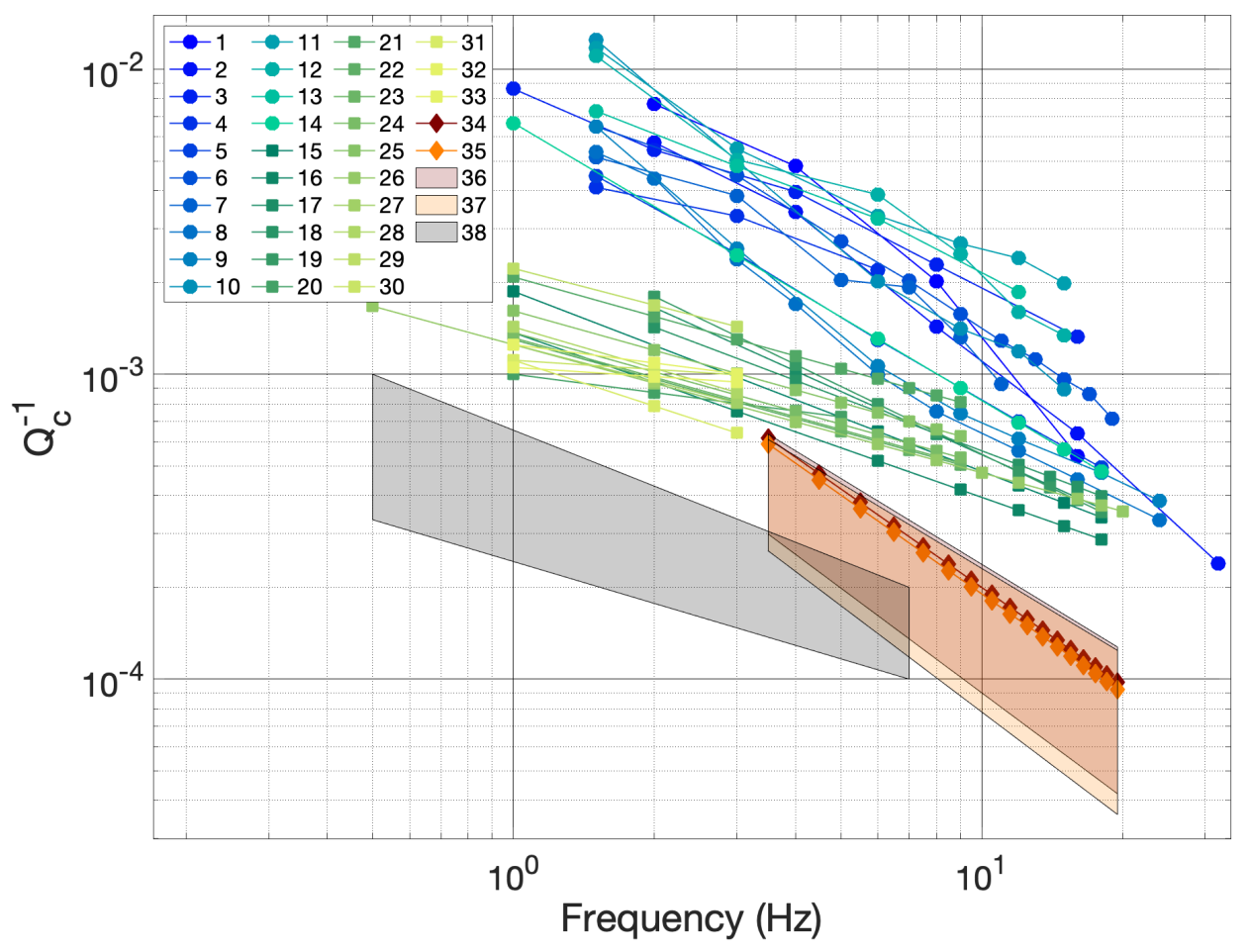

Figure 9: Coda attenuation $Q_{c}^{-1}$ as a function of the frequency for different regions on Earth (blue for tectonically active, green-yellow for cratons), the Moon (grey) and Mars (with $\alpha=0$ in red and $\alpha=1$ in orange). The numbers in inset refer to Table 2 where more details can be found on the geographical locations for Earth data, as well as the references of the original publications. 

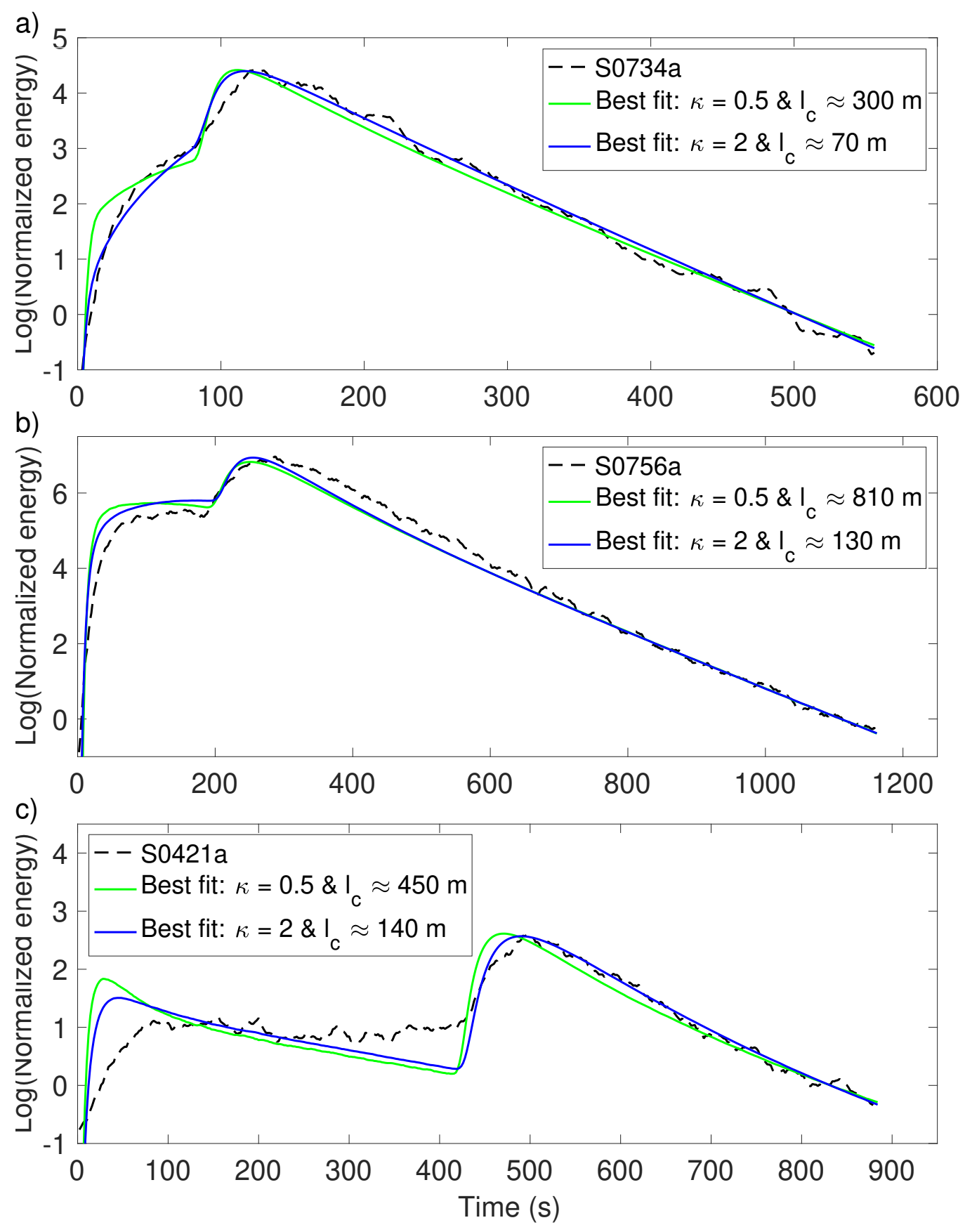

Figure 10: Radiative transfer modeling of the energy envelope of three VF events: (a) S0734a, (b) S0756a and (c) S0421a VF. The dashed black lines correspond to the energy envelope of the horizontal components filtered around $6 \mathrm{~Hz}$. The green and blue lines show the best-fitting radiative transfer models with $\kappa=0.5$ and $\kappa=2$ respectively. In these examples the $c^{p} / c^{s}$ ratio is set to 1.85 and $c^{s}=1.7 \mathrm{~km} / \mathrm{s}$. 
a)

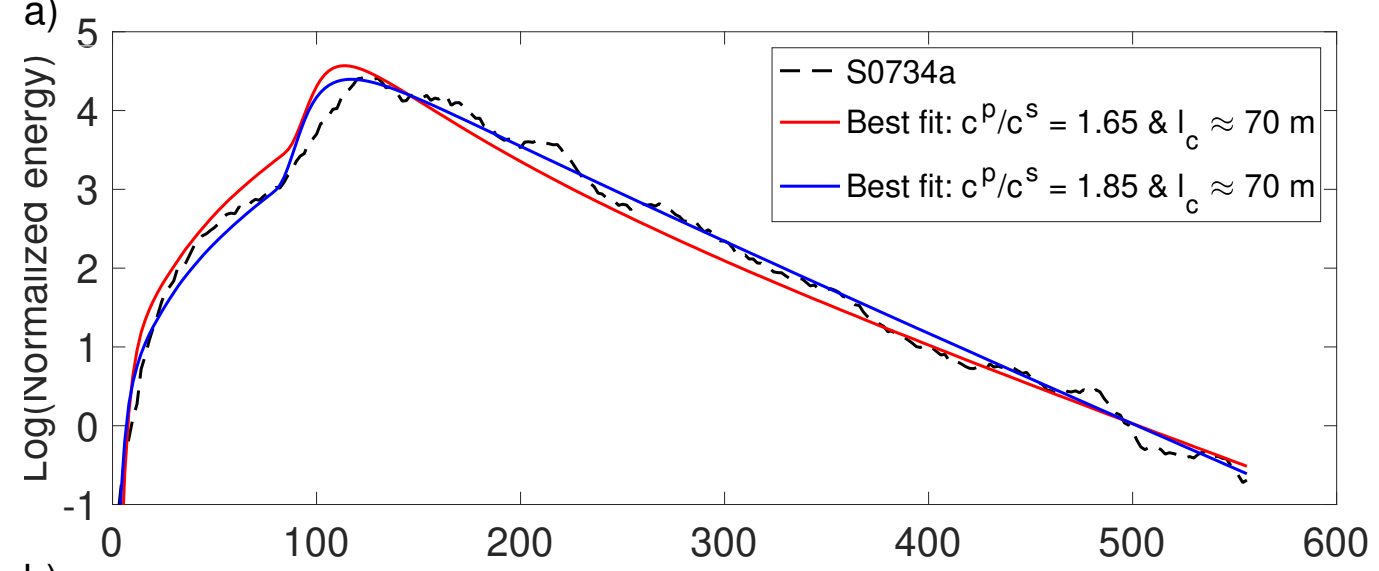

b)

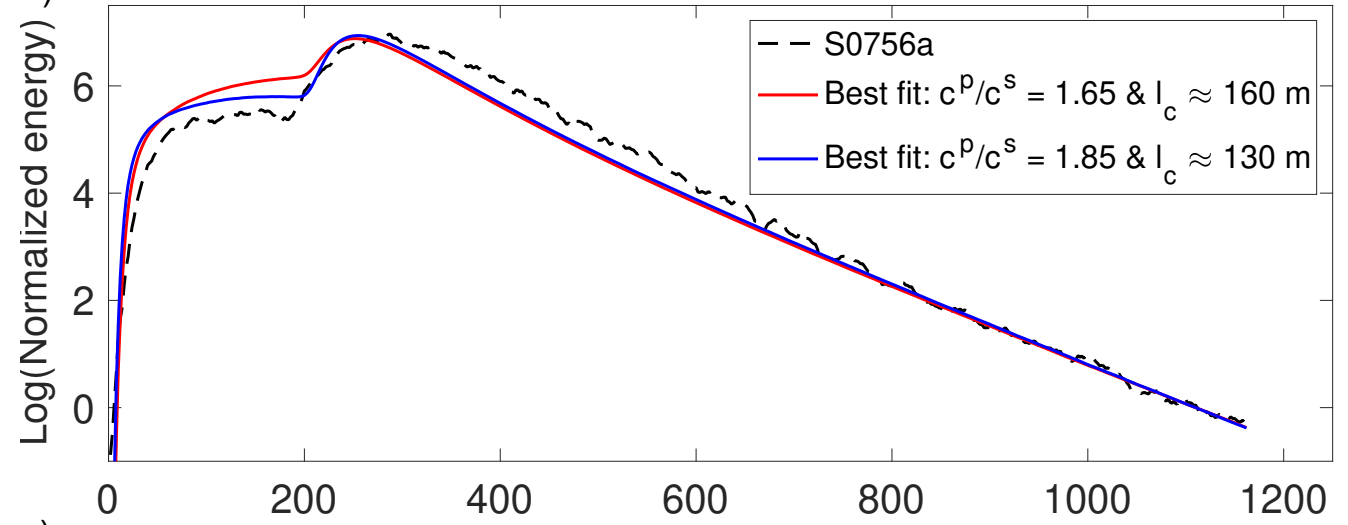

c)

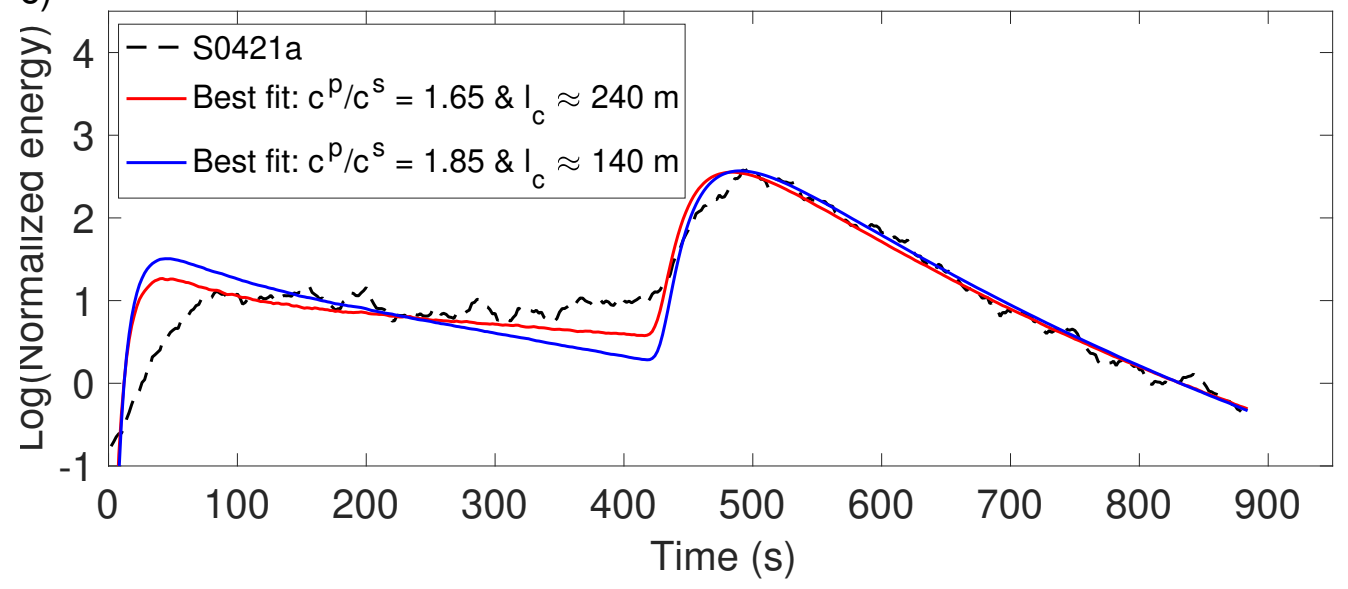

Figure 11: Radiative transfer modeling of the energy envelope of three VF events: (a) S0734a, (b) S0756a and (c) S0421a VF. The dashed black lines correspond to the energy envelope of the horizontal components filtered around $6 \mathrm{~Hz}$. The red and blue lines show the best-fitting radiative transfer models with $c^{p} / c^{s}=1.65$ and $c^{p} / c^{s}=1.85$, respectively. In these examples $\kappa$ is set to 2 and $c^{s}=1.7 \mathrm{~km} / \mathrm{s}$. 


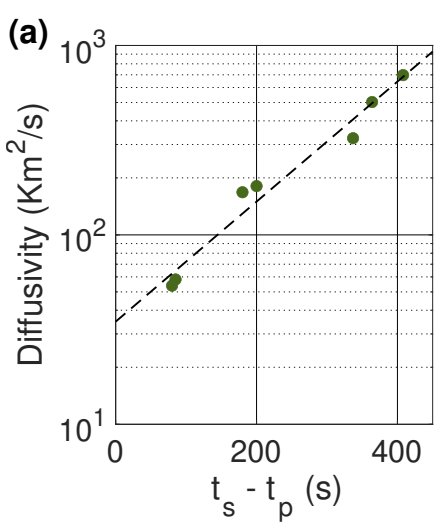

(b)

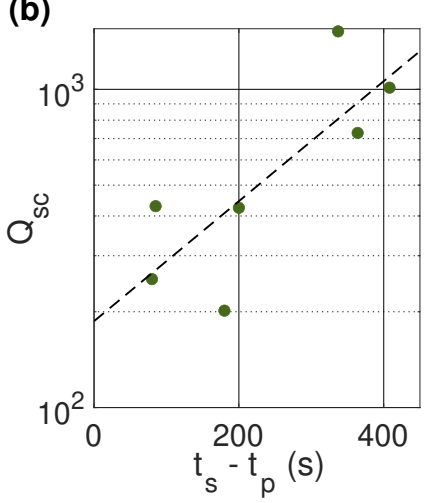

(c)

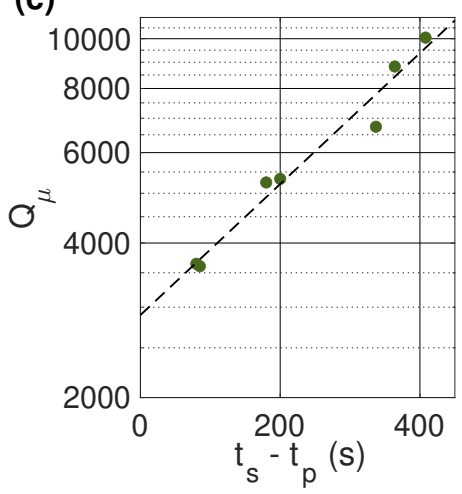

Figure 12: Inversion results for seven VF events. (a) Diffusivity $\left(\mathrm{km}^{2} / \mathrm{s}\right)$, (b) S-wave scattering quality factor and (c) S-wave absorption quality factor as a function of $\left(t_{s}-t_{p}\right)$ for selected VF events. The dashed line shows the best fit to the data assuming a law of the form $y=y_{0} \exp \left(t_{s}-t_{p}\right) / \xi$. 


\section{Appendices}

\section{A Envelope analysis of VF event S0734a using SP data}

In this appendix, we show the envelope characteristics of VF event S0734a as seen by the SP sensor in the $3-20 \mathrm{~Hz}$ frequency band. Figure A.1 complements Figure 3 presented in the main body of the text. For frequencies higher than $10 \mathrm{~Hz}$, we note some contamination of the envelopes by short transients (donks), which occur permanently and independent of seismic events (Ceylan et al., 2021). They entail fluctuations in the measurements of the time delay, as reflected in Figure A.1c. Data from the SP seismometer allow us to confirm the close to linear increase of the coda quality factor observed on the VBB, as well as the very weak frequency dependence of the peak delay time. We found $Q_{0}=476 \pm 9$ and $n=1.05 \pm 0.02$ for $\alpha=1$ ( $Q_{0}=431 \pm 7$ and $n=1.06 \pm 0.02$ for $\alpha=0$ ), which is in reasonable agreement with the measurements performed on the VBB in the $2.5-7.5 \mathrm{~Hz}$ band. 

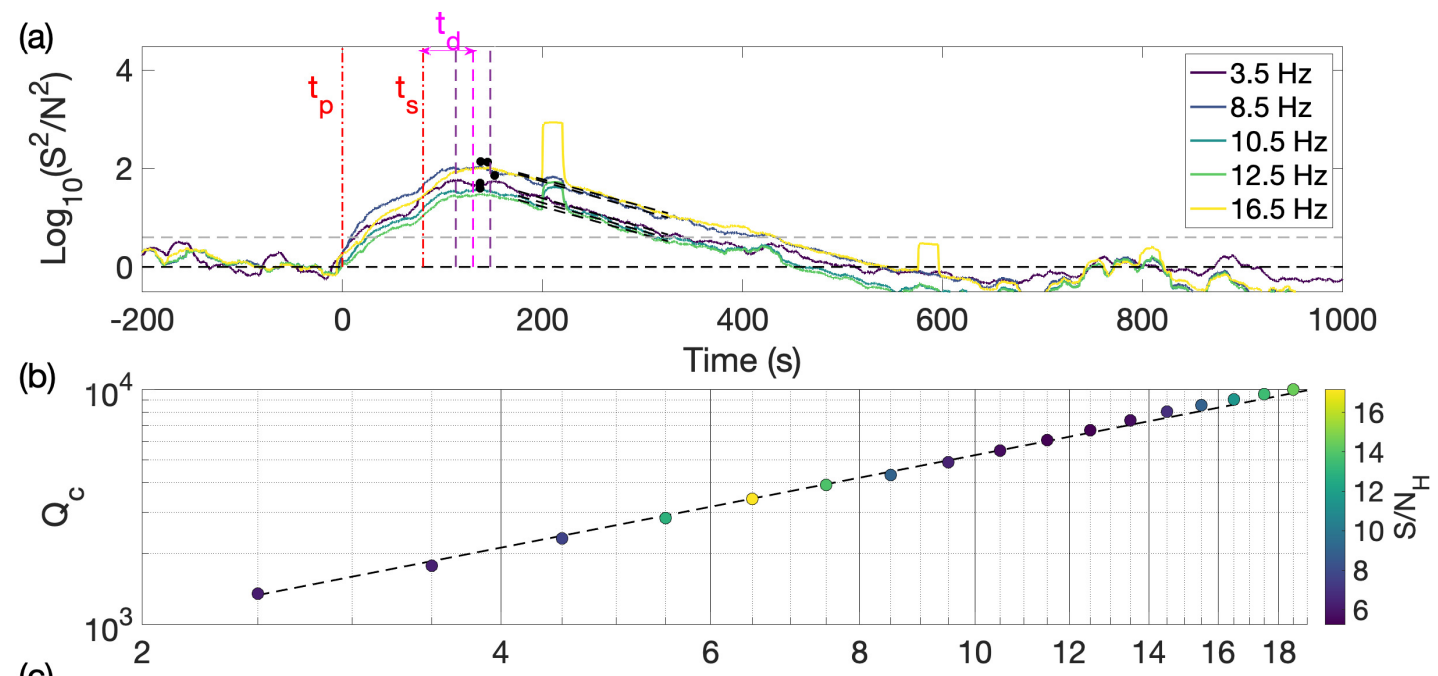

(c)

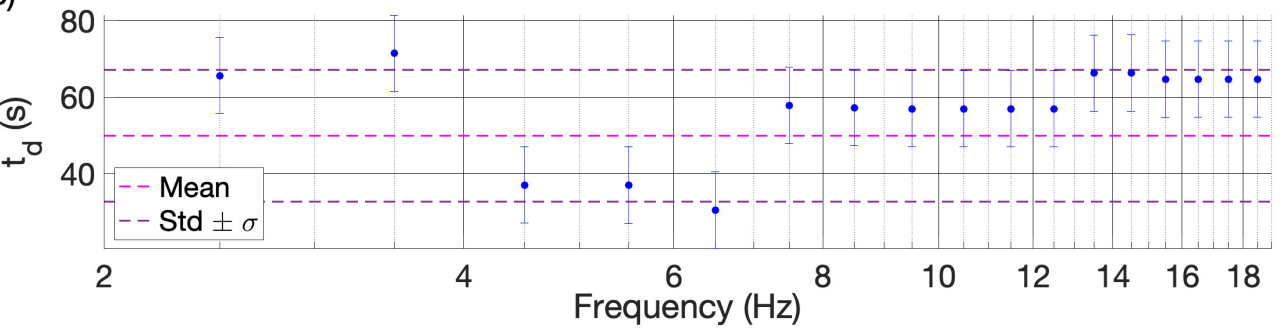

Figure A.1: Envelope characteristics of the VF event S0734a event. The data were collected by the SP sensors with sampling rates of 100 sps. (a) same as Figure 3. (b) same as Figure 3.b except for the power-law fit $Q_{c}=476 f^{1.05}$ ( $f$ is the frequency). (c) Same as Figure 3. 